Draft version SePtember 17, 2021

Typeset using LATEX twocolumn style in AASTeX63

\title{
SN 2018bsz: significant dust formation in a nearby superluminous supernova
}

T.-W. Chen (陳婷琬) ${ }^{1,2}$ S. J. Brennan, ${ }^{3}$ R. Wesson,${ }^{4}$ M. Fraser,${ }^{3}$ T. Schweyer, ${ }^{1}$ C. Inserra, ${ }^{5}$ S. Schulze, ${ }^{6}$ M. Nicholl,${ }^{7}$ J. P. Anderson,${ }^{8}$ E. Y. Hsiao (蕭亦麒) ${ }^{9}$ A. Jerkstrand,${ }^{1}$ E. Kankare, ${ }^{10}$ E. C. Kool, ${ }^{1}$ T. Kravtsov, ${ }^{10}$ H. Kuncarayakti,${ }^{10}{ }^{11}$ G. Leloudas, ${ }^{12}$ C.-J. Li (李傳睿) ${ }^{13}$ M. Matsuura, ${ }^{5}$ M. Pursiainen, ${ }^{12}$ R. Roy, ${ }^{14}$ A. J. Ruiter ${ }^{15}$ P. Schady, ${ }^{16}$ I. Seitenzahl, ${ }^{15}$ J. Sollerman, ${ }^{1}$ L. Tartaglia, ${ }^{17}$ L. Wang, ${ }^{18}$ R. M. Yates, ${ }^{19}$ S. YAng (杨圣),${ }^{1}$ D. BaAde, ${ }^{20}$ R. CARini, ${ }^{21}$ A. Gal-YAm ${ }^{22}$ L. Galbany ${ }^{23}$ S. GonzÁlez-Gaitán, ${ }^{24}$ M. Gromadzki, ${ }^{25}$ C. P. Gutiérrez, ${ }^{11,10}$ R. Kotak, ${ }^{26}$ K. Maguire, ${ }^{27}$ P. A. Mazzali, ${ }^{28,29}$ T. E. Müller-Bravo, ${ }^{30}$ E. Paraskeva, ${ }^{31,32}$ P. J. Pessis, ${ }^{33,8}$ G. Pignata, ${ }^{34,35}$ A. Rau, ${ }^{2}$ And D. R. YounG ${ }^{36}$

${ }^{1}$ The Oskar Klein Centre, Department of Astronomy, Stockholm University, AlbaNova, SE-10691 Stockholm, Sweden

${ }^{2}$ Max-Planck-Institut für Extraterrestrische Physik, Giessenbachstraße 1, 85748, Garching, Germany

${ }^{3}$ School of Physics, O'Brien Centre for Science North, University College Dublin, Belfield, Dublin 4, Ireland

${ }^{4}$ Department of Physics and Astronomy, University College London, United Kingdom

${ }^{5}$ School of Physics and Astronomy, Cardiff University, Queen's Building, The Parade, Cardiff, CF24 3AA, UK

${ }^{6}$ The Oskar Klein Centre, Department of Physics, Stockholm University, AlbaNova, SE-10691 Stockholm, Sweden

${ }^{7}$ Institute for Gravitational Wave Astronomy and School of Physics and Astronomy, University of Birmingham, Birmingham B15 2TT, $U K$

${ }^{8}$ European Southern Observatory, Alonso de Córdova 3107, Casilla 19, Santiago, Chile

${ }^{9}$ Department of Physics, Florida State University, 77 Chieftan Way, Tallahassee, FL 32306, USA

${ }^{10}$ Tuorla Observatory, Department of Physics and Astronomy, FI-20014 University of Turku, Finland

${ }^{11}$ Finnish Centre for Astronomy with ESO (FINCA), FI-20014 University of Turku, Finland

${ }^{12}$ DTU Space, National Space Institute, Technical University of Denmark, Elektrovej 327, 2800 Kgs. Lyngby, Denmark

${ }^{13}$ Institute of Astronomy and Astrophysics, Academia Sinica, No. 1, Sec. 4, Roosevelt Rd., Taipei 10617, Taiwan

${ }^{14}$ The Inter-University Centre for Astronomy and Astrophysics, Ganeshkhind, Pune-411007, India

${ }^{15}$ School of Science, The University of New South Wales, Australian Defence Force Academy, Canberra, ACT 2600, Australia

${ }^{16}$ Department of Physics, University of Bath, Claverton Down, Bath, BA2 7AY, UK

${ }^{17}$ INAF-Osservatorio Astronomico di Padova, Vicolo dell'Osservatorio 5, I-35122 Padova, Italy

${ }^{18}$ George P. and Cynthia Woods Mitchell Institute for Fundamental Physics $\&$ Astronomy, Texas A\&M University, Department of Physics and Astronomy, 4242 TAMU, College Station, TX 77843, USA

${ }^{19}$ Department of Physics, University of Surrey, Surrey GU2 7XH, UK

${ }^{20}$ European Organisation for Astronomical Research in the Southern Hemisphere (ESO), Karl-Schwarzschild-Str. 2, 85748 Garching b. M'unchen, Germany

${ }^{21}$ INAF - Osservatorio Astronomico di Roma, via Frascati 33, I-00078 Monte Porzio Catone, Italy

${ }^{22}$ Benoziyo Center for Astrophysics and the Helen Kimmel Center for Planetary Science, Weizmann Institute of Science, 76100 Rehovot, Israel

${ }^{23}$ Institute of Space Sciences (ICE, CSIC), Campus UAB, Carrer de Can Magrans, s/n, E-08193 Barcelona, Spain

${ }^{24}$ CENTRA, Instituto Superior Técnico, Universidade de Lisboa, Av. Rovisco Pais 1, 1049-001 Lisboa, Portugal

${ }^{25}$ Astronomical Observatory, University of Warsaw, Al. Ujazdowskie 4, 00-478 Warszawa, Poland

${ }^{26}$ Department of Physics \& Astronomy, University of Turku, Vesilinnantie 5, Turku FI-20014, Finland

${ }^{27}$ School of Physics, Trinity College Dublin, The University of Dublin, College Green, Dublin 2, Ireland

${ }^{28}$ Astrophysics Research Institute, Liverpool John Moores University, IC2, Liverpool Science Park, 146 Brownlow Hill, Liverpool L3 5RF, $U K$

${ }^{29}$ Max-Planck-Institut für Astrophysik, Karl-Schwarzschild Str. 1, D-85748 Garching, Germany

${ }^{30}$ School of Physics and Astronomy, University of Southampton, Southampton, Hampshire, SO17 1BJ, UK

${ }^{31}$ IAASARS, National Observatory of Athens, 15236, Penteli, Greece

${ }^{32}$ Department of Astrophysics, Astronomy Mechanics, Faculty of Physics, National and Kapodistrian University of Athens, 15784 Athens, Greece

${ }^{33}$ Facultad de Ciencias Astronómicas y Geofísicas (FCAG), Universidad Nacional de La Plata (UNLP), Paseo del bosque S/N, 1900, Argentina

${ }^{34}$ Departamento de Ciencias Fisicas, Universidad Andres Bello, Avda. Republica 252, Santiago, Chile 


\footnotetext{
${ }^{35}$ Millennium Institute of Astrophysics (MAS), Nuncio Monseñor Sotero Sanz 100, Providencia, Santiago, Chile ${ }^{36}$ Astrophysics Research Centre, School of Mathematics and Physics, Queen's University Belfast, Belfast BT7 1NN, UK
}

\section{ABSTRACT}

We investigate the thermal emission and extinction from dust associated with the nearby superluminous supernova (SLSN) 2018bsz. Our dataset has daily cadence and simultaneous optical and near-infrared coverage up to $\sim 100$ days, together with late time $(+1.7 \mathrm{yr})$ MIR observations. At 230 days after light curve peak the SN is not detected in the optical, but shows a surprisingly strong near-infrared excess, with $r-J>3$ mag and $r-K_{\mathrm{s}}>5$ mag. The time evolution of the infrared light curve enables us to investigate if the mid-infrared emission is from newly formed dust inside the SN ejecta, from a pre-existing circumstellar envelope, or interstellar material heated by the radiation from the SN. We find the latter two scenarios can be ruled out, and a scenario where new dust is forming in the SN ejecta at epochs $>200$ days can self-consistently reproduce the evolution of the SN flux. We can fit the spectral energy distribution well at $+230 \mathrm{~d}$ with $5 \times 10^{-4} M_{\odot}$ of carbon dust, increasing over the following several hundred days to $10^{-2} M_{\odot}$ by $+535 \mathrm{~d}$. SN $2018 \mathrm{bsz}$ is the first SLSN showing evidence for dust formation within the SN ejecta, and appears to form ten times more dust than normal core-collapse SNe at similar epochs. Together with their preference for low mass, low metallicity host galaxies, we suggest that SLSNe may be a significant contributor to dust formation in the early Universe.

Keywords: supernovae: general — supernovae: individual (SN 2018bsz)

\section{INTRODUCTION}

Core-collapse supernovae (CCSNe) are considered to be significant sites of cosmic dust production (Cernuschi et al. 1967; Hoyle \& Wickramasinghe 1970; for more recent reviews see Gall et al. 2011b; Sarangi et al. 2018b). Emission from newly formed dust has been seen in both CCSNe (e.g. Danziger et al. 1989; Lucy et al. 1989; Elmhamdi et al. 2004; Kotak et al. 2009; Matsuura et al. 2015) and in CCSN remnants (e.g. Douvion et al. 2001). Additional evidence for dust formation in CCSNe is that $\mathrm{CO}$ formation has been observed in near-infrared (NIR) spectra of Type II (hydrogen-rich) SNe (e.g. McGregor et al. 1987; Elias et al. 1988; Rho et al. 2018; Davis et al. 2019), and in Type Ic (hydrogen-poor) SNe (e.g. Rho et al. 2021). CO is a coolant for cooling the SN ejecta and can be regarded as a precursor to dust formation (e.g. Nozawa et al. 2003).

The amount of newly-formed dust in nearby CCSNe typically ranges between $10^{-5}$ and $10^{-3} M_{\odot}$ (see reviews by Gall et al. 2011b; Sarangi et al. 2018b), and is usually estimated from observations taken within three years after explosion, or in some cases even earlier. Dust masses inferred in SN remnants are generally larger, ranging between $10^{-2}$ and $0.1 M_{\odot}$ (e.g. Gomez et al. 2012; De Looze et al. 2017). Notably, Matsuura et al. (2011) used the Herschel Space Observatory to observe the farinfrared (FIR) emission of SN 1987A in 2010 and found that a large dust mass of $\sim 0.4-0.7 M_{\odot}$ was formed in this SN.
Observations of the most distant quasars $(z>6)$ show evidence for large masses of dust in galaxies in the early Universe (e.g. Bertoldi et al. 2003; Dwek et al. 2007). This is a strong argument for SNe being important dust producers in the high- $z$ Universe (e.g. Hirashita \& Ferrara 2002; Morgan \& Edmunds 2003; Maiolino et al. 2004; Bianchi \& Schneider 2007; Gall et al. 2011a; Calura et al. 2014), since other channels such as asymptotic giant branch (AGB) stars (through stellar outflows) will not have had sufficient time to produce a large quantity of dust at such early epochs (e.g. Dwek 1998; Ferrarotti \& Gail 2006; Dwek \& Cherchneff 2011). However, it has been suggested that the AGB stars may be an important contributor to dust formation by $z \sim 6-7$ (Valiante et al. 2009, 2011). Nonetheless, it is still a challenge to account for the large amount of dust in high- $z$ galaxies (e.g. Todini \& Ferrara 2001; Clayton et al. 2001; Dwek \& Cherchneff 2011), and additional dust production mechanisms are required (e.g. Matsuura et al. 2009).

Superluminous SNe (SLSNe) are massive stellar explosions which are 10-100 times brighter than normal CCSNe (Quimby et al. 2011; Gal-Yam 2019; Inserra 2019). SLSNe in general have higher explosion energies and more massive ejecta than normal CCSNe (e.g. Nicholl et al. 2015; Moriya et al. 2018), and are potentially very different sites for molecular and dust formation (c.f. Nozawa et al. 2003; Cherchneff \& Dwek 2009, 2010; Nozawa et al. 2011). SLSNe are suspected to be related to the first generation of stars in the Universe 
(Gal-Yam et al. 2009) and have so far been found at redshifts as high as $z \approx 2$ (Pan et al. 2017; Smith et al. 2018; Angus et al. 2019) or even $z \approx 4$ (Cooke et al. 2012; Moriya et al. 2019; Curtin et al. 2019).

In the local Universe $(z<2)$, the most common Type I (hydrogen-poor) SLSNe predominantly occur in dwarf galaxies with low stellar mass and low metallicity (Neill et al. 2011; Chen et al. 2013; Lunnan et al. 2015; Leloudas et al. 2015; Angus et al. 2016; Perley et al. 2016; Chen et al. 2017a; Schulze et al. 2018). Moreover, the dwarf galaxies hosting Type I SLSNe are often part of interacting systems (Chen et al. 2017b; Ørum et al. 2020). Such host galaxies are expected to be similar to galaxies at high redshift (e.g. Heckman et al. 2005; Cardamone et al. 2009; Bian et al. 2016). As Type I SLSNe potentially also have more massive progenitors (Jerkstrand et al. 2017; Mazzali et al. 2019), and lowmetallicity stars lose less mass before the SN explosion, they may be more effective at forming dust than normal CCSNe. Therefore, dust formation from Type I SLSNe could play an important role in the early formation and evolution of cosmic dust.

SN 2018bsz was classified as the closest Type I SLSN to date, at a redshift of $z=0.0267$ (Anderson et al. 2018). It exhibited several unusual features, including a long plateau prior to maximum light, and strong optical lines associated with C II. Late-time spectra show hydrogen, possibly indicating late-time interaction with an H-rich shell (e.g. Yan et al. 2015, 2017). Detailed analyses of extensive photometric (Roy et al. in prep.) and spectroscopic (Pursiainen et al. in prep.) datasets will be presented elsewhere.

In this paper, we focus on the late-time behaviour and mid-infrared (MIR) properties of SN 2018bsz, in order to investigate what role SLSNe play in cosmic dust formation. For consistency, we adopt the same basic parameters for SN 2018bsz as used in the early-phase study by Anderson et al. (2018). We hence assume a Hubble constant $H_{0}=73 \mathrm{~km} \mathrm{~s}^{-1} \mathrm{Mpc}^{-1}, \Omega_{\Lambda}=0.73$ and $\Omega_{\mathrm{M}}=0.27$ (Spergel et al. 2007). The corresponding luminosity distance $D_{L}=111 \mathrm{Mpc}$ and a distance modulus of $\mu=35.23 \pm 0.02 \mathrm{mag}$ are used for SN 2018bsz. The rest-frame phase is with respect to the $r$-band peak epoch $(\mathrm{MJD}=58267.5)$. Throughout this paper all magnitudes are reported in the $\mathrm{AB}$ system.

\section{OBSERVATIONS}

\subsection{Imaging from ultraviolet to mid-infrared wavelengths}

We obtained multi-band light curves for SN 2018bsz from the ultraviolet (UV) to the MIR using several telescopes. We used the UV/optical telescope
(UVOT; Roming et al. 2005) on board the Neil Gehrels Swift Observatory (Gehrels et al. 2004), under three target-of-opportunity programs ${ }^{1}$; the GammaRay Burst Optical/Near-Infrared Detector (GROND; Greiner et al. 2008) mounted on the 2.2-m MPG telescope at ESO's La Silla Observatory in Chile, as part of the GREAT survey (Chen et al. 2018); and the infrared spectrograph and imaging camera Son of ISAAC (SOFI) on the 3.58-m New Technology Telescope (NTT) in the framework of the extended Public ESO Spectroscopic Survey of Transient Objects (ePESSTO; Smartt et al. 2015) program. We were also awarded Director's Discretionary Time on the Spitzer Space Telescope (Werner et al. 2004) using the Infrared Array Camera (IRAC) instrument (Fazio et al. 2004) for late-time observations $^{2}$. In addition, archival mid-IR observations of the field of SN 2018bsz were taken by the Near Earth Object Wide-field Infrared Survey Explorer (NEOWISE; Mainzer et al. 2011) project using the Wide-field Infrared Survey Explorer (WISE; Wright et al. 2010), obtained as part of the NEOWISE Reactivation survey (NEOWISE-R; Mainzer et al. 2014).

We discuss the data reduction, photometric calibration and template subtraction for these images in detail in Appendix A. Photometry was measured on our optical and NIR images using the AutoPhOT code (Brennan et al. 2021). All magnitudes have been measured after subtraction of the background host galaxy. Figure 1 shows our assembled multi-band light curves for SN 2018bsz from 2 days after peak $(+2$ d) to +535 d, combined with the pre-peak ATLAS light curves from Anderson et al. (2018).

\subsection{Optical and NIR spectroscopy}

We also obtained a number of optical and NIR spectra for SN 2018bsz. At $+17 \mathrm{~d}$, we used the Spectrograph for INtegral Field Observations in the Near Infrared (SINFONI) instrument (Eisenhauer et al. 2003), mounted on the ESO 8-m Very Large Telescope (VLT), to observe SN 2018bsz and the surrounding H II regions. A spectrum at $+77 \mathrm{~d}$ was taken with the integral field unit (IFU) Wide-Field Spectrograph (WiFeS) (Dopita et al. 2007), mounted on the ANU 2.3-m telescope. A +108d spectrum was observed with the ESO Faint Object Spectrograph and Camera (EFOSC2) (Buzzoni et al. 1984) on the NTT through the ePESSTO collaboration. After the SN emerged from solar conjunction, we attempted a late-time observation with $\mathrm{X}$-Shooter, mounted on the VLT, between $+327 \mathrm{~d}$ and $+383 \mathrm{~d}$. No SN trace

\footnotetext{
${ }^{1}$ P.I.s Brown, Roy and Schulze

${ }^{2}$ Program ID: 14273, PI: Chen
} 


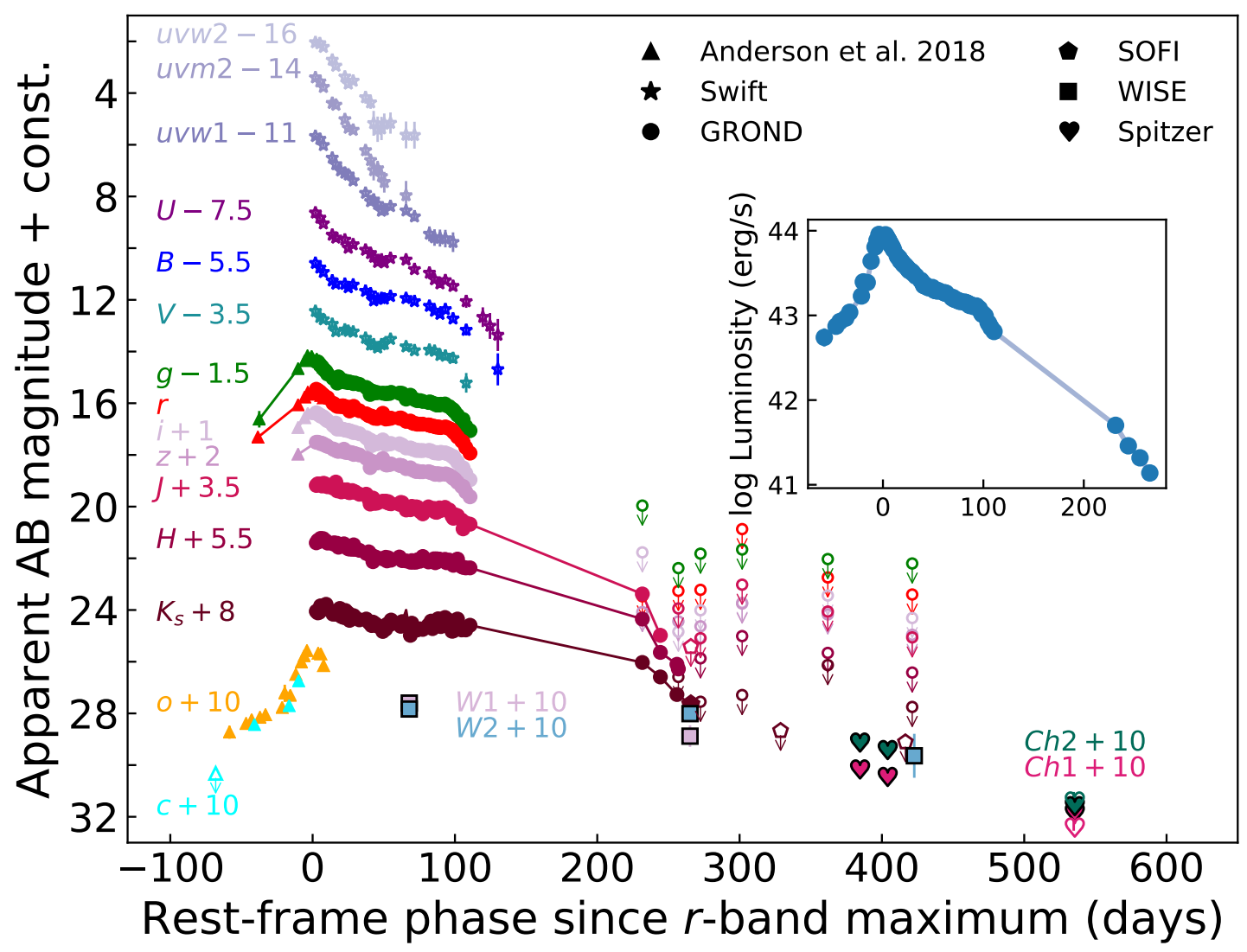

Figure 1. Multi-band, host-subtracted light curves of SN 2018bsz. Photometric measurements have not been corrected for Milky Way or host galaxy extinction. The pre-peak light curves are taken from Anderson et al. (2018). Marker shapes indicate data points taken using different facilities. The open symbols show $3 \sigma$ detection limits. The inset panel shows the bolometric light curve of SN 2018bsz (uncertainties are smaller than the point size). Note that at late-time phases ( $>200$ days), the SN was not detected in the optical but still detected in the NIR bands, with $r-K_{\mathrm{s}}>5$ mag.

was detected. Finally we used the Multi-Unit Spectroscopic Explorer (MUSE; Bacon et al. 2010) to observe the host-galaxy environment after the SN had faded at $+417 \mathrm{~d}$.

The instrumental configurations are listed in Table 1, and we describe the data reduction in detail in Appendix B. We show these optical spectra of SN 2018bsz in Fig. 2, while we plot the NIR spectra in Fig. A3. The complete set of spectra obtained for SN 2018bsz will be presented and analysed in Pursiainen et al. (in prep).

\section{OBSERVATIONAL CHARACTERISTICS OF SN 2018BSZ}

\subsection{Extinction}

Several sources can contribute to the extinction towards SN 2018bsz: a foreground (Milky Way) component; interstellar extinction within the host galaxy 2MASX J16093905-3203443; and (potentially) any circumstellar extinction surrounding the progenitor. The first of these components can be determined from Milky Way dust maps to be $E(B-V)_{\mathrm{MW}}=0.214 \mathrm{mag}$ (Schlafly \& Finkbeiner 2011). The latter two components are more problematic, and we consider a number of indirect approximate methods to measure them.

We extracted the host galaxy spectrum from our MUSE datacube at the position of SN 2018bsz using a circular shape aperture with $0^{\prime \prime} .6$ radius. After correcting for Milky Way extinction, we fitted and removed the stellar continuum using STARLIGHT (Cid Fernandes et al. 2005), and then measured the Balmer decrement in the spectrum. Assuming that the intrinsic value of the Balmer decrement of $\mathrm{H} \alpha / \mathrm{H} \beta$ is 2.86 (Osterbrock 1989), we found $E(B-V)_{\text {host }}$ to be $\sim 0.32 \mathrm{mag}$. The host of SN 2018bsz is part of an interacting galaxy pair, where in principle one may expect relatively high amounts of dust from active star formation (e.g. Lanz et al. 2013).

However, if we were to apply such a high extinction to the SN, the derived temperature of SN 2018bsz would 


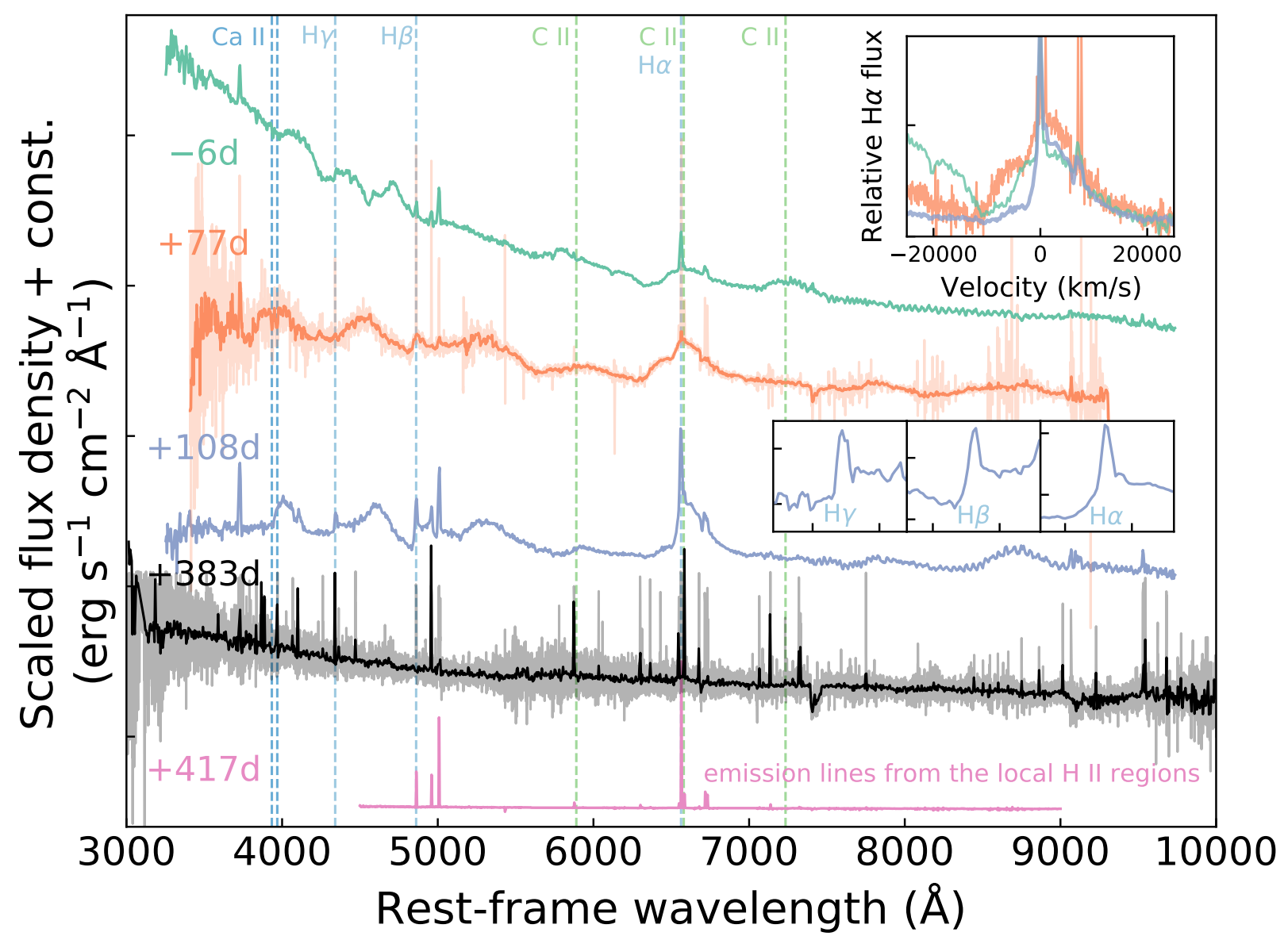

Figure 2. Spectroscopic evolution of SN 2018bsz. The -6d spectrum is taken from Anderson et al. (2018). The Balmer lines have emerged by $+77 \mathrm{~d}$ in our WiFeS spectrum (the darker line has been rebinned to $20 \AA$ ). Interestingly, a strong asymmetry in the line profiles of all Balmer lines (see lower inset panels for zoom) appears in the $+108 \mathrm{~d}$ EFOSC2 spectrum. No SN trace has been detection in the $+383 \mathrm{~d}$ X-Shooter spectrum (the darker line has been binned to $5 \AA$ ). The $+417 \mathrm{~d}$ spectrum is extracted from the MUSE cube at the SN position. After subtracting the stellar continuum, we highlight the strong narrow emission lines from the local H II regions (pink line). The upper inset shows a zoom in around $\mathrm{H} \alpha$, where the flux has been scaled to match the same continuum level on the red side of the line.

become $>18800 \mathrm{~K}$ assuming a blackbody at 3-20 days after the peak. The inferred temperature is higher than (by $\sim 5000 \mathrm{~K}$ ) and outside of the $3 \sigma$ temperature range of other Type I SLSNe at the same phase (see Fig. A1; Inserra et al. 2018b). We tested a range of different extinctions for SN 2018bsz and found that $E(B-V)_{\text {host }}$ between 0.03 and 0.10 mag can give a reasonable agreement with the temperature evolution of the other SLSNe at 3-20 days after the peak. Anderson et al. (2018) used $E(B-V)_{\text {host }}=0.04 \mathrm{mag}$ derived from the equivalent width of the sodium doublet (Na I D) lines (Poznanski et al. 2012). For consistency, we also adopt this value here, corresponding to host $A_{V}=0.13 \mathrm{mag}$ assuming $R_{V}=3.1$ (Cardelli et al. 1989). In addition, we also obtained a similar $A_{V}=0.15 \mathrm{mag}$ from our STARLIGHT best fit results. SN 2018bsz seems to lie towards the outskirts of its host and could well be positioned in the foreground, which may explain the relatively moderate reddening $\left(A_{V}=0.13 \mathrm{mag}\right)$ compared to that inferred from the Balmer decrement $\left(A_{V} \sim 1 \mathrm{mag}\right)$. If the Balmer decrement is measured over a large area containing multiple $\mathrm{H}$ II regions it will not necessarily be representative of the exact location of SN 2018bsz.

We note that that the presence of narrow emission lines at the location of SN 2018bsz in our MUSE spectrum suggests that those seen in the earlier SN spectra (Fig. 2) are from a local $\mathrm{H}$ II region.

\subsection{Light curve and bolometric light curve}


We have observational coverage for SN 2018bsz from UV, optical, NIR, and (for the first time for an SLSN) MIR wavelengths. Figure 1 shows our multi-band light curve for SN 2018bsz. With a daily cadence for more than 100 days, it makes SN 2018bsz the best monitored SLSN to date and it is also the first SLSN with daily contemporaneous 7-band coverage from optical to NIR, thanks to GROND (Fig. A2).

The early time evolution of SN 2018bsz was presented in Anderson et al. (2018), showing an $o$ band light curve with a long plateau slowly rising for $\sim 43$ days during the pre-maximum phase. The SN then displayed a steeper rise in magnitude for the next $\sim 18$ days in all optical bands. It reached a peak brightness at an absolute magnitude of $-20.3 \mathrm{mag}$ in the $r$ band. In this paper, we focus on the evolution after the peak, where the $r$-band light curve displays three phases. Initially, the SN faded rapidly for 18 days, before settling onto a long, slowlyfading plateau for 78 days. Finally it faded rapidly by $1 \mathrm{mag}$ in 15 days prior to the SN disappearing behind the Sun. The evolution was similar in the other optical bands, but with a shallower decline in the NIR. After the SN emerged from behind the Sun it was no longer detected at optical wavelengths, with $r>23.5 \mathrm{mag}$ on $+232 \mathrm{~d}$. At the same time, surprisingly, we found the SN was still detected in the NIR bands with $J \sim 19.9$, $H \sim 18.9$ and it was brightest in $K_{\mathrm{s}}$ with $\sim 18.0$ mag.

We constructed a bolometric light curve of SN 2018bsz (see the inset panel in Fig. 1) integrating over the full wavelength range from the UV to MIR. We have relatively sparse MIR coverage, hence we smoothly interpolate the MIR data between the epochs $+68 \mathrm{~d}$ and $+265 \mathrm{~d}$, and that although there is no MIR data before 68 days, the fractional MIR luminosity at $+68 \mathrm{~d}$ (only $1 \%$ ) suggests that the early time contribution of MIR is negligible. We describe in more detail the method used for constructing the bolometric light curve of SN 2018bsz and estimating the relative fractions of energy emitted in the different wavelength ranges in Appendix C.

After peak luminosity $\left(\sim 9 \times 10^{43} \mathrm{erg} \mathrm{s}^{-1}\right)$, the bolometric light curve declines rapidly for 16 days, by which time the luminosity has decreased by $\sim 50 \%$ (to $\sim$ $4.7 \times 10^{43} \mathrm{erg} \mathrm{s}^{-1}$ ). The bolometric light curve then continues to decline slowly; between $+17 \mathrm{~d}$ and $+40 \mathrm{~d}$, it drops another $\sim 50 \%$ in luminosity. Subsequently, from $+41 \mathrm{~d}$ to $+96 \mathrm{~d}$, SN 2018bsz further slows its decline rate, taking around twice as long to fade by another sim $50 \%$. At $+98 \mathrm{~d}$, the light curve shows a sudden drop in luminosity of roughly $40 \%$ in less than 13 days (to $\sim 6.5 \times 10^{42} \mathrm{erg} \mathrm{s}^{-1}$ ). Unfortunately our observing campaign was terminated at this epoch as SN 2018bsz went behind the Sun. When the SN became observable again at $+232 \mathrm{~d}$, it was 13 times fainter $\left(\sim 5 \times 10^{41} \mathrm{erg} \mathrm{s}^{-1}\right)$ than the last observation at $+111 \mathrm{~d}$. At late-time phases, there is no measurable contribution from the UV and optical to the luminosity, which is instead dominated by the NIR and MIR emission.

Using the same procedure as in Tartaglia et al. (2020), we investigated if there is a two-component spectral energy distribution (SED) by fitting the photometry at selected epochs $(+2.6,+50,+100$ and $+110 \mathrm{~d})$ with a blackbody function. We found that we do not require two distinct components at those epochs, and instead a single blackbody can fit the observed SED well (see Fig. A5). Table 2 lists the inferred blackbody temperature and radius evolution.

\subsection{Late-time Hydrogen from CSM}

At early phases, Anderson et al. (2018) suggested that no hydrogen was visible in the spectra of SN 2018bsz, and that the broad, strong feature at $\sim 6500 \AA$ (visible in Fig. 2) was not $\mathrm{H} \alpha$ but rather CII $\lambda 6580$ at a velocity of $-11000 \mathrm{~km} \mathrm{~s}^{-1}$. Anderson et al. argued that the absence of $\mathrm{H} \beta$ further strengthened the claim of SN 2018bz being hydrogen-poor. The presence of other Cir features at $5890,7234 \AA$ meant that carbon was a plausible identification for these lines. In our $+17 \mathrm{~d}$ NIR SINFONI spectrum, we see no broad SN emission at the wavelength of $\mathrm{Pa} \alpha$, confirming the lack of spectral signatures of $\mathrm{H}$ in the ejecta around maximum light.

By $+77 \mathrm{~d}$, however, it is clear that hydrogen is indeed present in the spectra of SN 2018bsz. At $+108 \mathrm{~d}$, a peculiar line profile characterised by a sharp cut off in flux in the blue is seen in $\mathrm{H} \alpha$, and an identical profile is also seen in $\mathrm{H} \beta$ and $\mathrm{H} \gamma$, making this a secure identification. We interpret the late-time appearance of hydrogen as a sign of interaction between the ejecta and H-rich circumstellar material (CSM).

Somewhat unusually, by $+108 \mathrm{~d}$ the Balmer line profiles are strongly asymmetric, with the blue side of the line being suppressed (Sec. 3.3). Those changes could be explained by dust formation in the post-shocked regions (and hence the absorption of the blue part of the line profiles). Alternatively, they may signify extraordinary kinematics or some extreme asymmetrical geometry in the ejecta.

The gas-phase metallicity in the local $\mathrm{H}$ II region is $\approx 0.3-0.4 Z_{\odot}$ as measured by strong-line methods (see Appendix B).

\subsection{Colour evolution and NIR excess}

During the first 75 days after maximum light, the colours of SN 2018bsz evolve slowly towards the red in most bands. If the steep optical decline around $+100 \mathrm{~d}$ 
was caused by extinction from such dust, then we might have expected substantial reddening to be observed in the $(g-r),(g-i)$ and $(g-z)$ colour evolution between $+98 \mathrm{~d}$ and $+111 \mathrm{~d}$. However, we see no strong colour changes as a function of wavelength during this period, with $(g-r) \sim 0.4 \Rightarrow 0.4,(g-i) \sim 0.2 \Rightarrow 0.2$ and $(g-z) \sim 0.3 \Rightarrow 0.4$. Similarly, the appearance of asymmetric Balmer profiles (Sec. 3.3) does not coincide with any strong change in the colours of SN $2018 \mathrm{bsz}$.

At $+108 \mathrm{~d}$, the spectrum of SN 2018bsz appears to be becoming optically thin (e.g. we see the disappearance of the absorption component in Ca). We hence consider that the contemporaneous luminosity drop in all optical bands is most likely due to the ejecta transitioning to the nebular phase. Accompanying this, we see a slight redward colour evolution, similar to what is seen in other $\mathrm{SNe}$ as they become nebular (for example in SN 2004et; Maguire et al. 2010). As for the earlier phases, we do not require any dust component to explain this evolution.

After the SN emerged from solar conjunction, it was not detected in deep imaging in the optical bands, but was still visible in the NIR bands. At $+232 \mathrm{~d}$, the colours were $r-J>3.1, r-H>4.1$ and $r-K_{\mathrm{s}}>4.9 \mathrm{mag}$ (the magnitudes are corrected for the Milky Way and the host galaxy dust extinction). Moreover, the SN shows a prominent $K_{\mathrm{s}}$-band excess (see Fig. A6). We measured a colour $J-K_{\mathrm{s}}=1.73 \pm 0.21 \mathrm{mag}$ at $+232 \mathrm{~d}$, and an even redder colour $J-K_{\mathrm{s}}=2.77 \pm 0.30 \mathrm{mag}$ at $+244 \mathrm{~d}$. The SOFI photometry at $+266 \mathrm{~d}$ (with a colour $J-K_{\mathrm{s}}>$ $2.15 \mathrm{mag}$ ) confirms this $K_{\mathrm{s}}$-band excess from GROND.

This rapid evolution to red colours suggests either that new dust is condensing in the SN ejecta, or that there is an IR light echo from pre-existing circumstellar dust or interstellar material heated by the SN flash. We investigate those scenarios in detail in Sec. 4.

\section{DUST MODELLING RESULTS}

Infrared emission from CCSNe may arise from new dust forming in the ejecta, a thermal echo from CSM, or a thermal echo from nearby interstellar material (ISM). The origin of the emission (which could be from more than one of these mechanisms) may be inferred from the temporal evolution of the emission. New dust will form at a temperature close to the dust sublimation temperature, and if it is heated by the decay of radioactive elements in the ejecta, it will fade exponentially. In the case of a SLSN powered by the spin down of a newly formed magnetar (Kasen \& Bildsten 2010), the energy deposited in the expanding remnant would similarly be expected to decline quickly. On the other hand, flashheated circumstellar or interstellar dust will in general have a temperature lower than the sublimation temper- ature of the constituent dust. The temporal evolution of a light echo is determined by the geometry of the echoing material. Extensive CSM may give rise to very long-lasting echoes, such as those seen to continue many years after the explosions of SN 2002hh (Barlow et al. 2005; Meikle et al. 2006) and SN 1980K (Sugerman et al. 2012).

In the following we expand on each of these scenarios in more detail.

\subsection{Interstellar echoes}

We model the evolution of the SED of SN 2018bsz using the radiative transfer code MOCASSIN (Ercolano et al. 2003, 2005) ${ }^{3}$.

We calculated the predicted emission from interstellar and circumstellar echoes using MOCASSIN's option to sum emission from within ellipsoidal regions defined by light-travel time (see Wesson et al. 2010 for a description). This approach is valid if photons are subject to a single scattering only and hence requires that the echoing medium be optically thin.

Figure 4.1 shows the temporal evolution of the emission from a slab of dust measuring $10 \mathrm{pc} \times 10 \mathrm{pc} \times 1 \mathrm{pc}$, $1 \mathrm{pc}$ in front of SN 2018bsz, containing $1 \mathrm{M}_{\odot}$ of amorphous carbon dust. We modelled the ISM echo using a light pulse of 75 days, with an integrated energy of $5.5 \times 10^{49} \mathrm{erg}$ and a temperature of $10^{4} \mathrm{~K}$. We find that the IR emission from this slab is several orders of magnitude below the observed fluxes at early times, and its flux is almost constant with time. Placing the slab closer to the SN does not increase the echo fluxes significantly as the echoing region then becomes smaller. A higher flash luminosity or dust mass may be possible but nevertheless, an ISM echo is unlikely to be a significant contributor to the evolving IR emission, except possibly at much later epochs than considered here.

\subsection{Circumstellar echoes}

Dense CSM close to the SN may give rise to a significant thermal echo. We constructed models of CSM echoes by assuming a smooth shell in which the density is proportional to $\mathrm{r}^{-2}$, with the echo calculated using the same flash parameters as the ISM models. The temporal evolution of the echo is determined by the inner and outer radii, with the echo ending once the epoch exceeds twice the light-travel time to the outer radius. We calculated the evolution of a light echo from shells of both carbon and silicate dust, with grain radii of 0.01 , 0.1 and $1.0 \mu \mathrm{m}$. We considered shells with combinations of inner radii between 0.001 and $0.1 \mathrm{pc}$ and outer radii

\footnotetext{
${ }^{3}$ We used version 2.02 .73 of the code.
} 

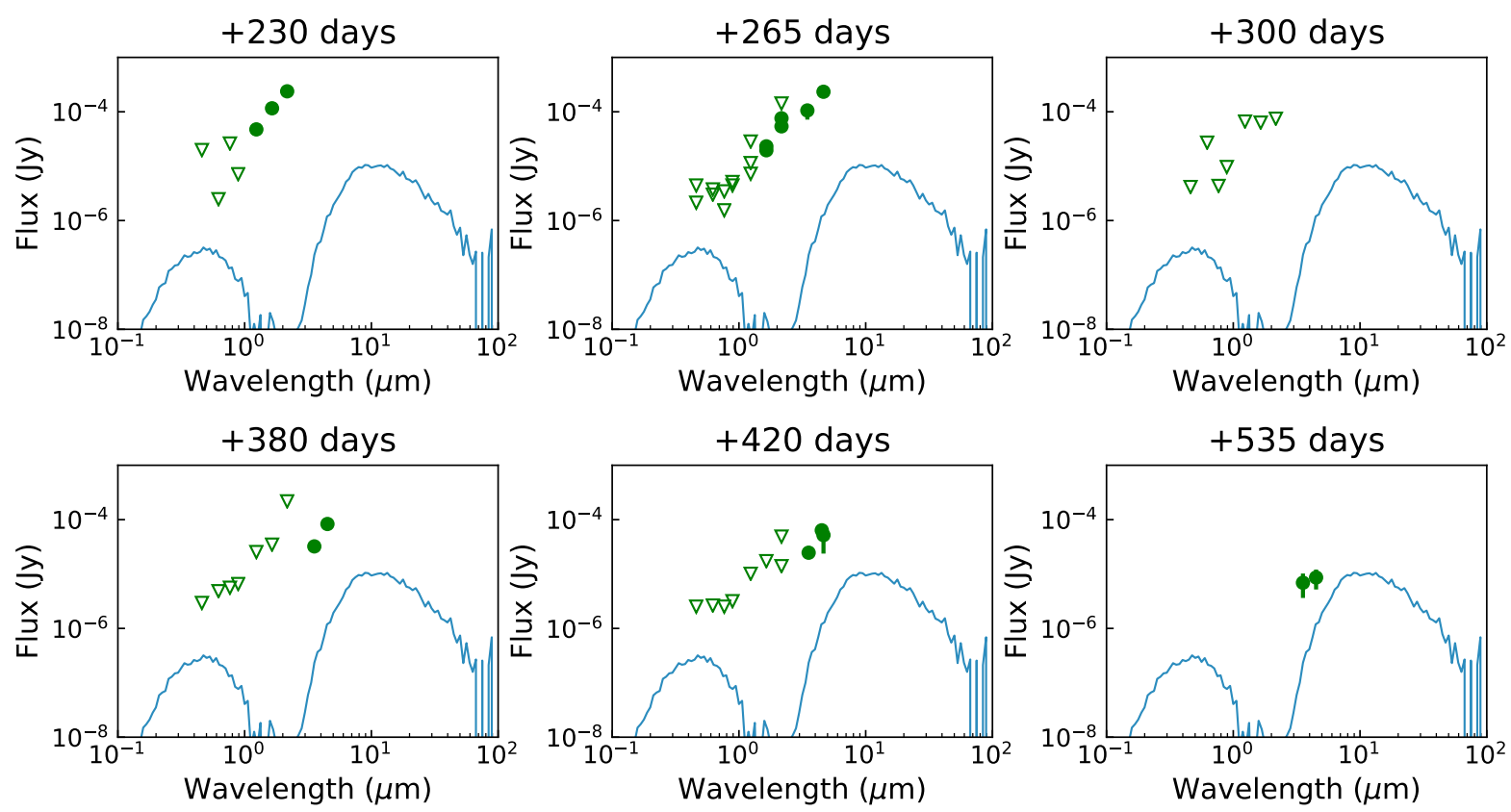

Figure 3. The temporal evolution of the emission from an ISM slab of dust. The IR flux from this ISM slab model is orders of magnitude below the observations, and its flux is quite constant over time. The green circles are the Milky Way extinction corrected fluxes of SN 2018bsz, and empty triangles denote $3 \sigma$ upper limits where we have a non-detection. The predicted SN SED is shown by the blue curves and the assumed dust model is described in Sec. 4.1.

between 0.03 and 0.5 pc. We are unable to find a shell geometry that can account for the evolution of the SED, for three reasons:

Firstly, the temporal evolution is not reproduced. For there to be a CSM echo lasting until $+535 \mathrm{~d}$, the shell needs to be very extensive, with at least some material $\sim 270$ light days $(0.23 \mathrm{pc})$ behind the $\mathrm{SN}$. This gives rise to a slowly-declining echo, from which the fluxes at $+230 \mathrm{~d}$ and $+265 \mathrm{~d}$, for example, would not be significantly different. The observations show that over these 35 days, the IR fluxes in fact decline significantly.

Secondly, the flash-heated dust is too cool to reproduce the SED.

And thirdly, the optical non-detections at $+230 \mathrm{~d}$ and $+265 \mathrm{~d}$ cannot be reproduced in most of these echo scenarios; models that predict IR fluxes of the same order as those observed also generally predict detectable optical signals from scattered light. Only models with very small grains can reproduce the optical non-detections. Silicate dust does not absorb enough radiation to reemit significantly in the IR.

Figure 4.2 shows the predicted echo flux from a CSM extending from 0.1 to $0.25 \mathrm{pc}$ from the $\mathrm{SN}$, for both $0.01 \mu \mathrm{m}$ and $0.1 \mu \mathrm{m}$ grains. This geometry gives IR fluxes which are reasonably close to the observations, but for either grain size, the echo gives too little flux at early times and too much at late times, and the larger grains over-predict the optical fluxes.

Due to the lack of observational constraints, we have not considered echoes from an asymmetricallydistributed CSM. An asymmetric CSM could give rise to thermal echoes with a temporal evolution more consistent with the rapid fading of the IR emission. However, it remains difficult to see how the optical non-detections at $+230 \mathrm{~d}$ and $+265 \mathrm{~d}$ could be reproduced in this scenario.

\subsection{New dust forming in the $S N$ ejecta}

Given that an ISM echo cannot contribute significantly to the IR fluxes, and an echo from a symmetric CSM is not compatible with the evolution of the SED, we consider models of newly-forming dust in the ejecta. We adopt a configuration in which the heating source is distributed throughout an expanding dusty shell, in which the dust density is proportional to $\mathrm{r}^{-2}$. We adopt a temperature of $5,000 \mathrm{~K}$ for the heating source, although this is not strongly constrained by the observations, given the optical non-detections. We assumed a velocity of $1000 \mathrm{~km} \mathrm{~s}^{-1}$ at the inner edge of the ejecta, and an outer radius 5 times the inner radius. The predicted SEDs are not strongly sensitive to the adopted inner radius, but outer radii much larger than the adopted value result in dust too cool to match the observed SED. 

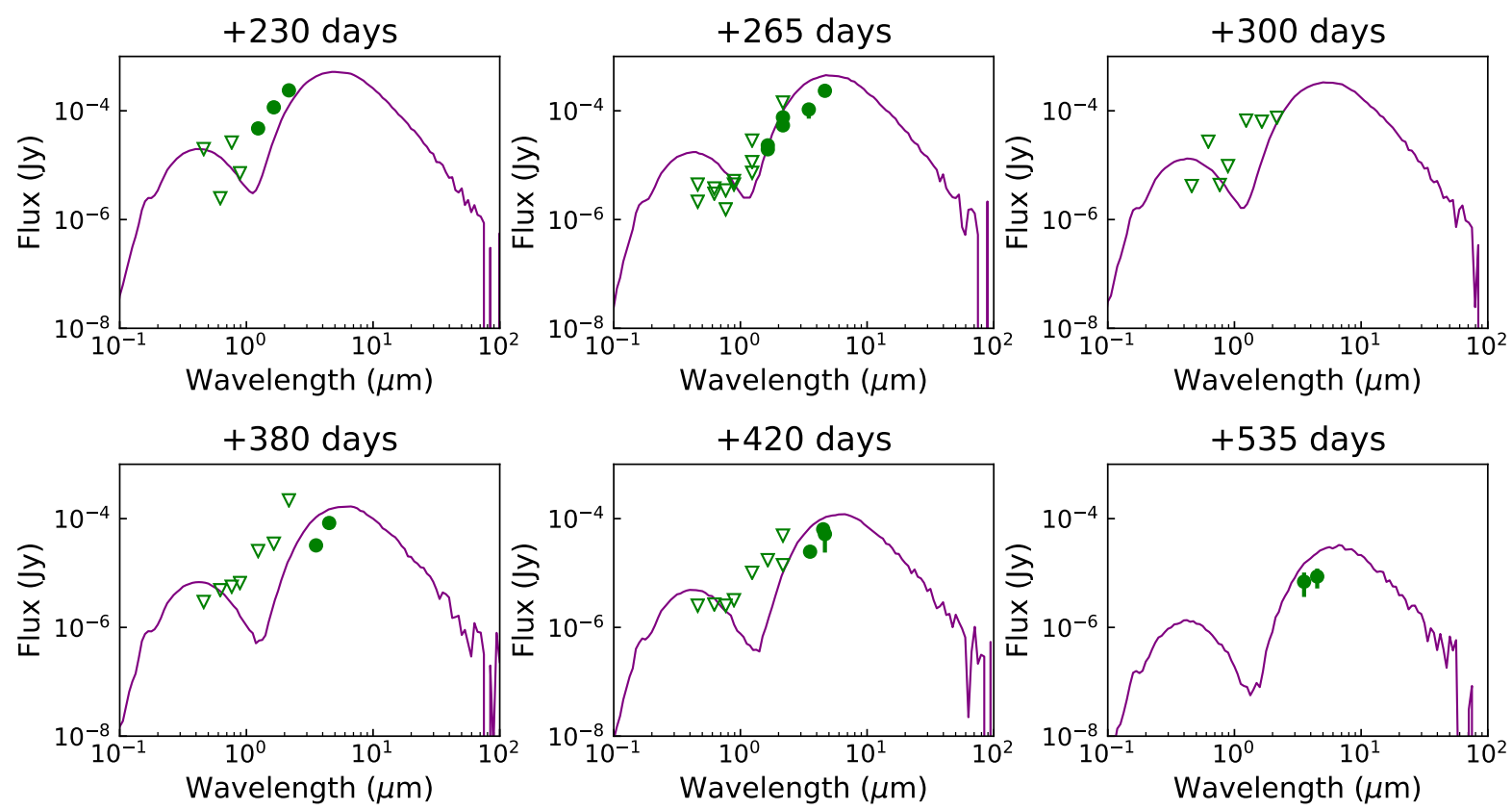

Figure 4. Predicted SED evolution from the smooth CSM shell, with grain sizes of $0.1 \mu \mathrm{m}$. The symbols are the same as that used in Fig. 3. The assumed dust model is described in Sec. 4.2.

We first constructed models for +230 d. At this epoch, we find that silicate dust would evaporate unless the grains are larger than $1 \mu \mathrm{m}$ in size. Much smaller grains of amorphous carbon dust can survive and their formation at this early epoch is more plausible. We therefore assume that the dust is composed only of amorphous carbon.

While Rayleigh-Taylor instabilities are expected to form during the SN explosions and give rise to clumpy ejecta distributions (e.g. Arnett et al. 1989), the optical non-detections are difficult to reconcile with a clumpy dust distribution. In the case of SN 1987A, clumpy dust models have found that a clump volume filling factor of around 0.1 is required (Ercolano et al. 2007; Wesson et al. 2015; Bevan \& Barlow 2016). If this is adopted for SN 2018bsz, optical emission exceeding the observed non-detections is predicted. We therefore investigated clumpy dust distributions with much higher volume filling factors, and find that a filling factor greater than about 0.7 is required to match the optical non-detections. For a given total mass, the predicted SEDs for smooth dust distributions are not significantly different to the predictions for clumpy distributions filling so much of the volume, and so for simplicity, we adopt a smooth dust distribution. With this assumption, we find that the $+230 \mathrm{~d}$ SED can be well fitted by $5 \times 10^{-4} \mathrm{M}_{\odot}$ of amorphous carbon dust with a grain size of $0.1 \mu \mathrm{m}$.
At days $+230 \mathrm{~d}$ and $+265 \mathrm{~d}$, we use the luminosity from the bolometric light curve (Fig. A4) in our models. To fit subsequent epochs, we investigated different rates of decline of the luminosity. From its peak until $+265 \mathrm{~d}$, the bolometric light curve declines with a half-life of approximately 32 days, much faster than the half-life of ${ }^{56} \mathrm{Co}$. However, extrapolating this to $+535 \mathrm{~d}$, we find that the luminosity would then be much too low to reproduce the observed IR fluxes. To constrain the luminosity at this epoch, we consider two limiting cases. We first estimate an upper limit to the luminosity of $1.7 \times 10^{41} \mathrm{erg} \mathrm{s}^{-1}$; above this, the emission from a $5000 \mathrm{~K}$ blackbody would exceed the observed infrared fluxes. We then estimate a lower limit by considering models containing $10^{-3} \mathrm{M}_{\odot}$ of dust, which maximises the emission at 3.6 and $4.5 \mu \mathrm{m}$ (higher/lower dust masses give predicted SEDs which peak at longer/shorter wavelengths). To match the observed fluxes at these wavelengths with this dust mass requires a luminosity of about $1.7 \times 10^{40} \mathrm{erg} \mathrm{s}^{-1}$.

We therefore fitted subsequent epochs by expanding the modelled dusty shell, reducing the luminosity according to ${ }^{56} \mathrm{Co}$ radioactive decay, and then varying the dust mass uniformly throughout the shell if necessary. We note that while the late time light curve of SN 2018bsz is probably not powered by Ni (e.g. Inserra et al. 2013), the decline rate of SLSNe is quite similar to that of ${ }^{56}$ Co until 600 days (Moriya et al. 2017), mak- 


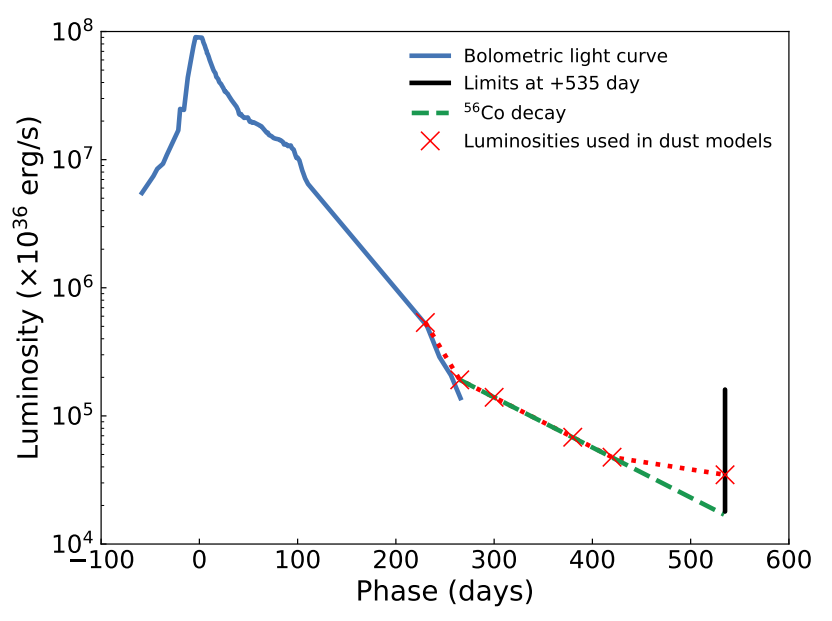

Figure 5. The observed bolometric light curve of SN 2018bsz and late time extrapolations. Since we only detect SN 2018bsz in the MIR at $+535 \mathrm{~d}$, we must estimate the true bolometric luminosity that serves at input to our dust models. The lower limit on the late time luminosity (lower edge of the black vertical line) comes from the requirement that the modeled dust mass does not decrease from previous epochs. The upper limit (upper edge of the black vertical line) comes from the most luminous 5,000K blackbody fit which matches the MIR points. The green dashed line shows the ${ }^{56}$ Co decay, while the orange points show the final luminosities that we use in our dust modeling.

ing this a reasonable choice of function for the input luminosity.

We find that until $+535 \mathrm{~d}$, models with a steadily increasing dust mass can fit the observations. At $+535 \mathrm{~d}$, calculating the luminosity by extrapolating the ${ }^{56} \mathrm{Co}$ decay, we are able to fit the observed IR fluxes but with a lower dust mass than at $+420 \mathrm{~d}$. Requiring that the dust mass does not decrease, we find that a higher luminosity is necessary to fit the MIR emission. Figure 4.3 shows the bolometric light curve, the extrapolated ${ }^{56} \mathrm{Co}$ decline, and the actual luminosities used in our models.

Our predicted SEDs are shown in Fig. 4.3. Our models require that the dust mass increases from about $5 \times 10^{-4} \mathrm{M}_{\odot}$ at $+230 \mathrm{~d}$ to $10^{-2} \mathrm{M}_{\odot}$ at $+535 \mathrm{~d}$. This pattern of the dust mass increasing steadily over a few hundred days is consistent with the pattern of dust formation observationally determined in other CCSNe (e.g. Wesson et al. 2015; Bevan \& Barlow 2016; Bevan et al. 2020). In Fig. 4.3 we plot literature values of estimated dust masses at different epochs of other CCSNe (between $10^{2}$ and $10^{4}$ days) together with our estimated masses for the dust in SN 2018bsz. At all epochs, the largest source of uncertainty in the estimate of the dust mass is the adopted luminosity. At +230 and $+265 \mathrm{~d}$, the bolometric luminosity is well-constrained, and the resulting uncertainty in the dust mass is about a factor of 2. At $+300 \mathrm{~d}$ where only upper limits to the fluxes are available, the dust mass is unconstrained. At later epochs when the luminosity is extrapolated, the uncertainties are larger. At $+535 \mathrm{~d}$, as described above, a dust mass of zero is nominally compatible with the observations but would require a much higher luminosity than at earlier epochs. To estimate the uncertainty on the dust mass at this epoch, we require that the luminosity is not higher than at previous epochs, and then vary both the dust mass and luminosity to obtain fits to the SED. In this way we estimate that the dust mass uncertainty is about a factor of five. The possible contribution of a CSM echo at this epoch may introduce an additional uncertainty, although if we assume that the mass of newly-formed ejecta dust does not decrease at any point, the overestimate in the dust mass that would be caused by attributing a CSM echo to newly-formed dust emission must be small.

These uncertainties notwithstanding, SN 2018bsz appears to have formed more dust at these epochs than most other CCSNe. The shape of the dust growth curve of SN 2018bsz is very similar to the general trend seen in Fig. 4.3, and SN 2018bsz clearly lies at the upper edge of the spread seen in normal CCSNe. We fit the evolution of SN 2018bsz and all other CCSNe separately using $\mathrm{M}_{\text {dust }} \propto t^{2.4}$ (adopting from the SN 2010jl ejecta dust evolution from Gall et al. 2014), and find that SN 2018bsz to have formed ten times more dust than normal CCSNe at similar epochs. In the case of SN 1987A, Wesson et al. (2015) found that the majority of the dust present 23 years after the explosion must have formed at epochs later than 1000 days. If a similar formation process is occurring in SN 2018bsz, the $10^{-2} \mathrm{M}_{\odot}$ of dust present at $+535 \mathrm{~d}$ will be a small fraction of the final dust mass.

\section{DISCUSSIONS AND CONCLUSION}

Several lines of evidence suggest that new dust forming in the ejecta of SN 2018bsz is responsible for the observed IR excess after $\sim 200$ days. CSM echoes cannot reproduce the temporal evolution of SN 2018bsz; while the CSM geometries that could explain the IR emission at much later times ( $>230$ days) would also predict detectable optical emission from scattered light, which we do not observe. ISM echoes, on the other hand, would have to be from extremely dense dust clouds close to the SN to give rise to detectable IR emission. We find that a slab of dust $1 \mathrm{pc}$ in front of the SN containing $1 \mathrm{M}_{\odot}$ of dust would result in an echo at $535 \mathrm{~d}$ with fluxes about two orders of magnitude below those ob- 

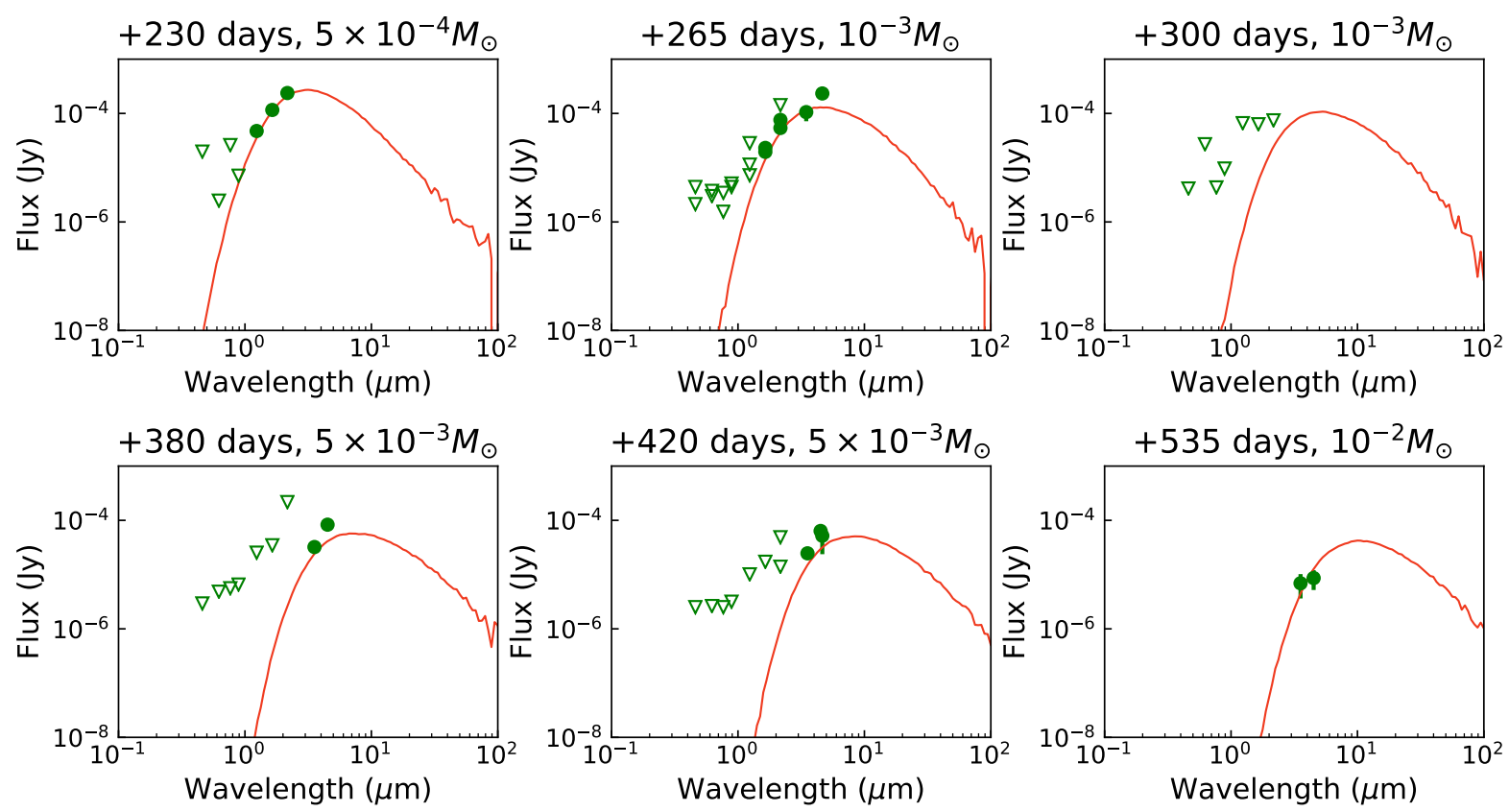

Figure 6. Predicted emission from new dust in the SN ejecta at epochs from $+230 \mathrm{~d}$ to $+535 \mathrm{~d}$ after the peak. The symbols are the same as those used in Fig. 3. The assumed dust model is described in Sec. 4.3.

served. Therefore, our modelling suggest newly-formed dust in the SN ejecta at epochs $>200$ days, with a dust composition of pure amorphous carbon. We estimate that the dust mass formed in SN 2018bsz is about ten times larger than what is found for other $\mathrm{SNe}$ at similar phases, with the dust mass increasing from about $5 \times 10^{-4} \mathrm{M}_{\odot}$ at $+230 \mathrm{~d}$ to $10^{-2} \mathrm{M}_{\odot}$ at $+535 \mathrm{~d}$.

The fact that the SN 2018bsz was hydrogen poor at early times (Anderson et al. 2018), and that the Balmer lines appear after 77 days, before dust formation (after 200 days), suggests that the dust formation is driven by the interaction between the SN ejecta and a hydrogenrich CSM. Mixing of metal-rich material in the ejecta with a hydrogen-rich CSM can lead to the formation of large amounts of dust, as the necessary molecular processes are thought to be more efficient in a hydrogenrich environment (Rawlings \& Williams 1990).

Late-time CSM interaction, together with a strongly asymmetric ejecta (as seen, for example in like Eta Carinae) may also account for the peculiar Balmer line profiles. In this scenario, around 100 days after peak brightness we observe the Balmer lines first forming, and later when the SN ejecta sweeps up the CSM it causes the observed changes in their profiles. The blue side of the line profiles are absorbed by swept-up CSM, while thanks to the asymmetric geometry, we can still see the red side of the lines. We do not see such profiles in the metal lines, likely because they come from deeper layers in the ejecta. Dust will form as the shocked, swept-up CSM cools, as is observed at late times ( $>200$ days).

Although SN 2018bsz is the first SLSN without hydrogen lines at maximum light for which dust has been detected, a late-time NIR excess was observed in one Type II SLSN, SN 2008es ( $M_{V} \sim-22 \mathrm{mag}$ ) (Gezari et al. 2009; Miller et al. 2009; Inserra et al. 2018b). It has a broad $\mathrm{H} \alpha$ feature during the photospheric phase and does not show any sign of strong interaction between SN ejecta and circumstellar shells in the early spectra. Bhirombhakdi et al. (2019) obtained late-time photometry of SN 2008es, which showed $r-K^{\prime}>1.5 \mathrm{mag}$ around 250-300 days after the explosion. The authors suggested that this NIR excess indicates dust condensation in a cool dense shell that formed through circumstellar interaction (e.g. Mattila et al. 2008). We note however that SN 2018bsz has a much redder colour of $r-K_{\mathrm{s}}>4.9 \mathrm{mag}$ at a similar epoch to SN 2008es.

Omand et al. (2019) investigated the effect of a pulsar wind nebula on dust formation and found that the pulsar wind can accelerate or delay dust formation time. The formation timescale of carbon dust can vary from $\sim 180$ to $\sim 590$ days for Type I SLSN assuming a magnetar power source. In our case for SN 2018bsz we detect carbon dust formation within one year after explosion, which is consistent with their predicted timescale.

Type I SLSNe are rare events, with about one SLSN for every $3500_{-720}^{+2800}$ CCSNe estimated from the Palomar 


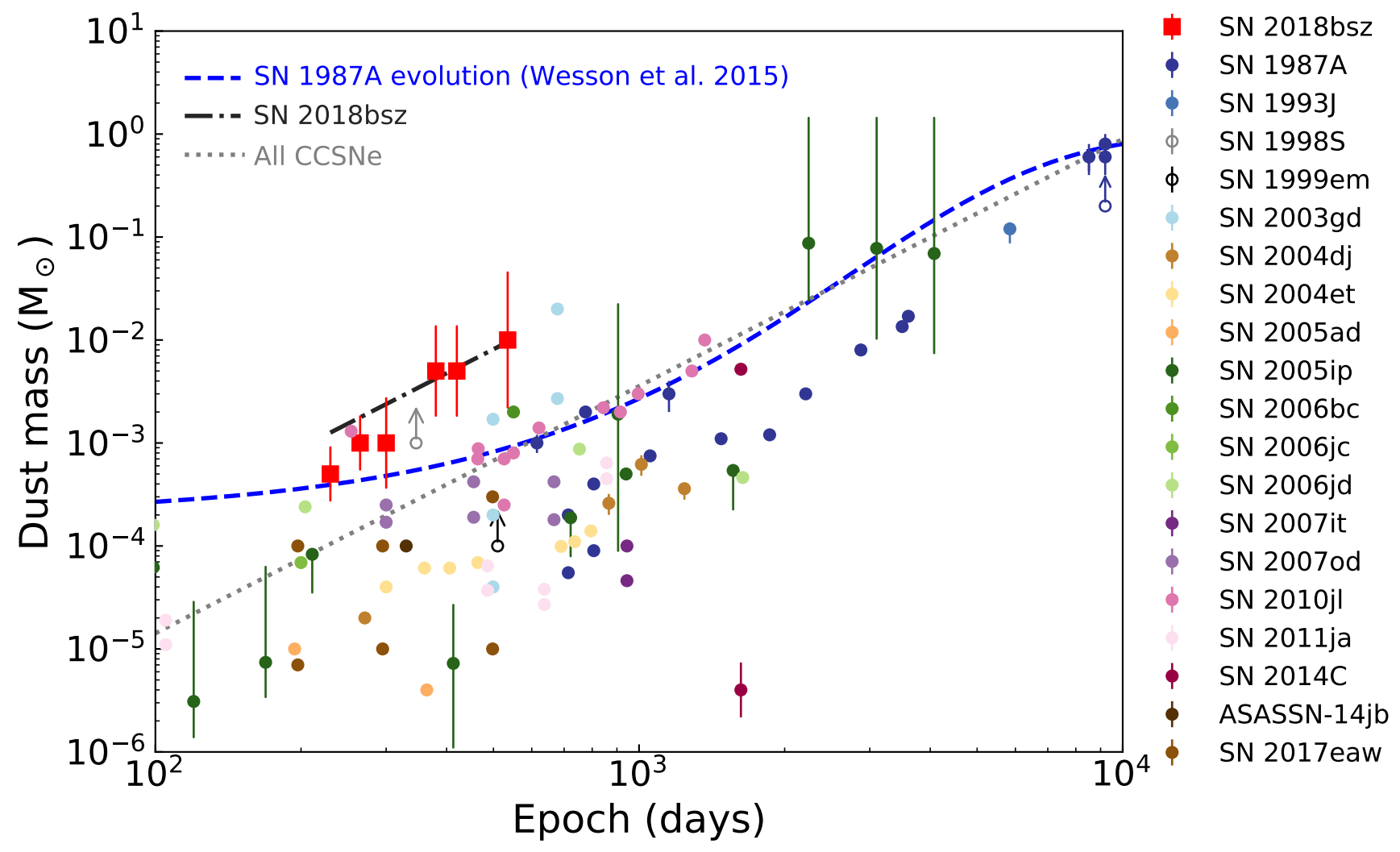

Figure 7. The dust masses inferred for SN 2018bsz, compared with literature values for other CCSNe. We find that SN 2018bsz formed ten times more dust than normal core-collapse SNe at similar epochs. We fit the evolution of SN $2018 \mathrm{bsz}$ and all other CCSNe separately, using $\mathrm{M}_{\text {dust }} \propto t^{2.4}$ (adopting from the SN 2010jl ejecta dust evolution from Gall et al. 2014), and show the evolution in black dash-dot and grey dotted lines, respectively. The blue dashed line shows the evolution of the dust mass in SN 1987A inferred by Wesson et al. (2015). See the webpage ${ }^{a}$ for the full collection; the references we used in this figure are

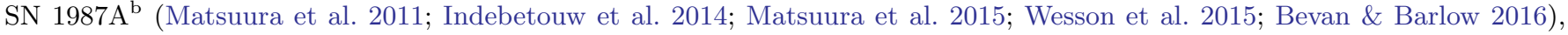
SN 1993J (Bevan et al. 2017), SN 1998S (Pozzo et al. 2004), SN 1999em (Elmhamdi et al. 2003), SN 2003gd (Sugerman et al. 2006; Meikle et al. 2007), SN 2004dj (Szalai et al. 2011), SN 2004et (Kotak et al. 2009), SN 2005ad and SN 2005af (Szalai \& Vinkó 2013), SN 2005ip (Fox et al. 2010; Stritzinger et al. 2012; Bevan et al. 2019), SN 2006bc (Gallagher et al. 2012), SN 2006jc (Sakon et al. 2009), SN 2006jd (Stritzinger et al. 2012), SN 2007it (Andrews et al. 2011), SN 2007od (Andrews et al. 2010), SN 2010j1 ${ }^{c}$ (Maeda et al. 2013; Sarangi et al. 2018a; Bevan et al. 2020), SN 2011ja (Andrews et al. 2016), SN 2014C (Tinyanont et al. 2019a), ASASSN-14jb (Meza et al. 2019), and SN 2017eaw (Tinyanont et al. 2019b).

a: https://www.nebulousresearch.org/dustmasses/

b: We note that Dwek \& Arendt (2015) report high dust masses at early epochs for SN 1987A. However, we do not plot these here as they are incompatible with the observed gradual evolution of the emission-line profiles (Wesson \& Bevan, in prep. 2021).

c: For SN 2010jl we show the dust masses assuming carbon dust in Sarangi et al. (2018a).

Transient Factory sample by Frohmaier et al. (2021). These authors found that the relative rate was higher in low-mass $\left(\log \mathrm{M}<9.5 \mathrm{M}_{\odot}\right)$ galaxies, with one SLSN for every $1700_{-720}^{+1800}$ CCSNe. Furthermore, sub-solar metallicity appears to be required for massive stars to explode as Type I SLSNe (Perley et al. 2016; Chen et al. 2017b; Schulze et al. 2018). Such low-mass, lowmetallicity galaxies are common in the early Universe (e.g. Heckman et al. 2005; Cardamone et al. 2009; Bian et al. 2016). The dust mass deduced for SN 2018bsz is $\sim 10$ times larger than what is seen in most CCSNe. If the rate of Type I SLSNe were $10^{-2}$ of the CCSN rate in the high- $z$ Universe, they could thus produce up to $10 \%$ of the dust in the early Universe. In fact, one could even speculate that if SLSNe are produced by more massive stars than CCSNe, and if the initial mass function at high redshift is top heavy, then SLSNe could be even more common.

A more self-consistent (although still approximate) estimate of the dust mass produced by Type I SLSNe at high redshift can be obtained from cosmological galaxy evolution simulations. For example, the cosmic dust production rate density (DD) from SLSNe can be calculated from the L-GALAXIES 2020 semi-analytic model 
(Henriques et al. 2020; Yates et al. 2021). We assume that (a) Type I SLSNe only form in galaxies with gas-phase metallicity $\leq 0.5 \mathrm{Z}_{\odot}$, (b) the initial mass function (IMF) is fixed over time and space, (c) SLSNe produce 10 times as much dust per event as CCSNe, and (d) the shape of the DD evolution from SNe follows that of the cosmic star formation rate density (SFRD). Under these assumptions, and following the dust evolution modelling implemented by Vijayan et al. (2019), L-Galaxies 2020 predicts an approximate DD of $-7.56 \lesssim \log _{10}\left(M_{\text {dust,SLSN }} / \mathrm{M}_{\odot} \mathrm{yr}^{-1}\right.$ $\left.\mathrm{Mpc}^{-3}\right) \lesssim-6.99$ at $z=7$, depending on the simulation box (i.e. mass resolution) and the ISM enrichment efficiency chosen. This is around 2 orders of magnitude lower than the DD expected from CCSNe at $z=7$ (see Vijayan et al. 2019, fig. 8). However, it is roughly comparable to the expected contribution from AGB stars at this redshift, which may play an important role (see Valiante et al. 2009; Mancini et al. 2015). This indicates that Type I SLSN are indeed a non-negligible contributor to dust production in the high redshift Universe.

We caution that these estimates assume that all SLSNe produce as much dust as SN 2018bsz. However, SN 2018bsz is a somewhat unusual SLSN and appears quite different to the bulk of the Type I SLSN population (according to its unique light curve-shape and spectroscopic evolution). It is hence quite possible that only a few percent of the SLSN population produce dust.

Observational data for Type I SLSNe remains the limiting factor in knowing their dust masses. We may well be systematically missing the window to see evidence for dust-formation in SLSNe, as the majority of them are found at $z>0.1$ and do not have rest-frame $K$-band observations, especially at late times ( $>200$ days). In fact, only two other Type I SLSNe (among $\sim 200$ events) have late-time rest-frame K-band data, namely PTF12dam (Nicholl et al. 2013; Chen et al. 2015; Vreeswijk et al. 2017), and SN 2015bn (Nicholl et al. 2016)).

Finally, a high luminosity combined with their preference for dwarf hosts, makes Type I SLSNe appealing targets for high-redshift (young Universe) searches, e.g. with Euclid (Inserra et al. 2018a), the James Webb Space Telescope (JWST) or the Rubin Observatory Legacy Survey of Space and Time (LSST) (Inserra et al. 2021). JWST in particular can reach down to 27 th magnitude in the infrared (IR), potentially allowing for observations of SLSN out to $z=20$ (Wang et al. 2019), and spectroscopic classification of some of these up to $z \sim 5$ (Nicholl et al. 2020). Further systematic observations by JWST will help to determine how much dust is formed in typical Type I SLSNe.

\section{ACKNOWLEDGMENTS}

We thank Prof. Michael J. Barlow for his valuable discussions and inputs about ejecta dust and circumstellar dust. We would also like to thank Margherita Molaro for valuable discussions on the analytic estimation of dust masses at high redshift. T.-W. C. thanks to Sijie Chen, Dan Perley, Jinyi Shangguan, Lin Yan, Jason Spyromilio, Stefan Taubenberger, Seppo Mattila and John Danziger for useful discussions of this object.

T.-W. C. acknowledges the funding from the Alexander von Humboldt Foundation and the EU Funding under Marie Skłodowska-Curie grant H2020-MSCA-IF2018-842471. S. J. B acknowledges support from Science Foundation Ireland and the Royal Society (RSEA/3471). RW acknowledges support from European Research Council (ERC) Advanced Grant 694520 SNDUST (PI: Barlow). AJR and IRS acknowledge financial support from the Australian Research Council under grants FT170100243 and FT160100028, respectively. MF is supported by a Royal Society - Science Foundation Ireland University Research Fellowship. E. Y. H. acknowledges the support provided by the National Science Foundation under Grant No. AST-1613472 and by the Florida Space Grant Consortium. C.-J.L. is supported by the Taiwanese Ministry of Science and Technology grant MOST 109-2112-M001-040 and 109-2811-M-001-545. MN is supported by a Royal Astronomical Society Research Fellowship and by the European Research Council (ERC) under the European Union's Horizon 2020 research and innovation programme (grant agreement No. 948381). GL was supported by a research grant (19054) from VILLUM FONDEN. AGY's research is supported by the EU via ERC grant No. 725161, the ISF GW excellence center, an IMOS space infrastructure grant and BSF/Transformative and GIF grants, as well as The Benoziyo Endowment Fund for the Advancement of Science, the Deloro Institute for Advanced Research in Space and Optics, The Veronika A. Rabl Physics Discretionary Fund, Minerva, Yeda-Sela and the Schwartz/Reisman Collaborative Science Program; AGY is the recipient of the Helen and Martin Kimmel Award for Innovative Investigation. TMB was funded by the CONICYT PFCHA / DOCTORADOBECAS CHILE/2017-72180113. L.G. acknowledges financial support from the Spanish Ministry of Science, Innovation and Universities (MICIU) under the 2019 Ramón y Cajal program RYC2019-027683 and from the Spanish MICIU project PID2020-115253GA-I00. MG is supported by the EU Horizon 2020 research and innovation programme under grant agreement No 
101004719. S.G.G. acknowledges support by FCT under Project CRISP PTDC/FIS-AST-31546/2017 and UIDB/00099/2020. KM is funded by the EU H2020 ERC grant no. 758638. G.P. is supported by ANID Millennium Science Initiative - ICN12_009.

GROND observations at La Silla were performed as part of the programs 102.A-9099 and 103.A-9099. Part of the funding for GROND (both hardware as well as personnel) was generously granted from the LeibnizPrize to Prof. G. Hasinger (DFG grant HA 1850/281). This work is based (in part) on observations collected at the European Organisation for Astronomical Research in the Southern Hemisphere, Chile as part of ePESSTO and ePESSTO+, (the Public ESO Spectroscopic Survey for Transient Objects Survey), ESO programs 199.D-0143 and 1103.D-0328. We also collect data from the ESO Science Archive Facility using the SINFONI instrument under the ESO program 2101.D-5026, the X-Shooter instrument under the ESO program 0103.D-0697 and the MUSE instrument under the ESO program 0103.D-0523. This work is based [in part] on observations made with the Spitzer Space Telescope, which was operated by the Jet Propulsion Laboratory, California Institute of Technology under a contract with NASA, Spitzer Observing program ID: 14273. This publication makes use of data products from the Widefield Infrared Survey Explorer, which is a joint project of the University of California, Los Angeles, and the Jet Propulsion Laboratory/California Institute of Technology, funded by the National Aeronautics and Space Administration. We acknowledge the use of public data from the Neil Gehrels Swift Observatory data archive, under the target-of-opportunity programs.

The STARLIGHT project is supported by the Brazilian agencies CNPq, CAPES and FAPESP and by the France-Brazil CAPES/Cofecub program. IRAF is distributed by the National Optical Astronomy Observatory, which is operated by the Association of Universities for Research in Astronomy (AURA) under a cooperative agreement with the National Science Foundation. This research made use of PYPEIT, a Python package for semi-automated reduction of astronomical slit-based spectroscopy. 


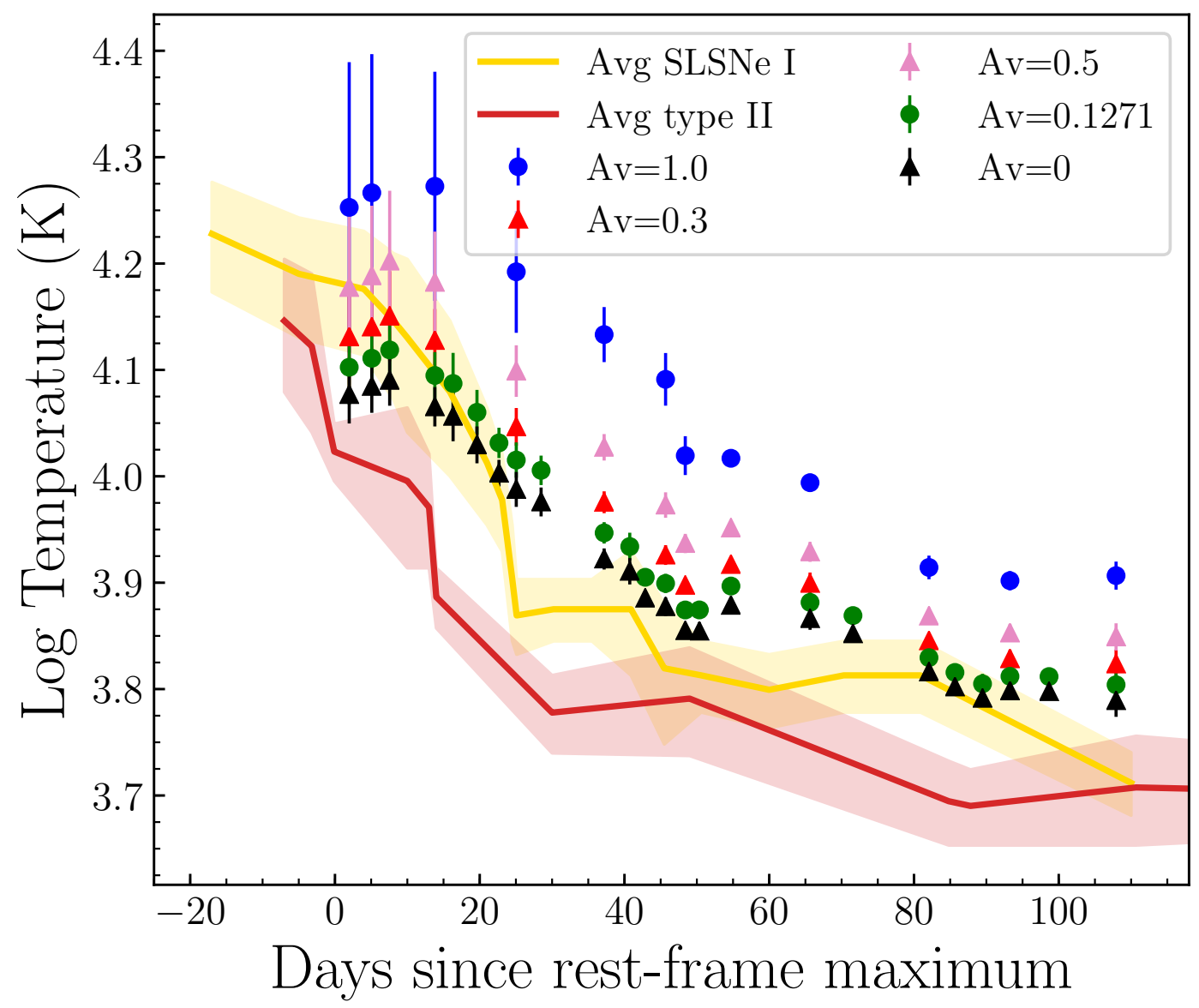

Figure A1. Temperature evolution of SN 2018bsz in comparison to the average evolution of Type I SLSNe and Type II SNe (Inserra et al. 2018b). The comparison sample comprises 14 Type I SLSNe (Inserra et al. 2013; Nicholl et al. 2015) and 30 Type II SNe (Elmhamdi et al. 2003; Maguire et al. 2010; Inserra et al. 2011, 2012; Botticella et al. 2010; Tomasella et al. 2013; Gutiérrez et al. 2017). The shaded regions represent the 99.73 percent confidence ranges for temperature of each class of SNe. The temperature of SN 2018bsz is measured from SED fits assuming a blackbody at selected epochs. We find that if the host extinction is between $A_{V}=0.1$ and 0.3 mag we obtain reasonable agreement with the SLSNe at 3-20 days after the peak.

\section{APPENDIX}

\section{A. IMAGING OBSERVATIONS OF SN 2018BSZ}

\section{A.1. UVOT observations with Swift}

We obtained 28 epochs of photometry for SN 2018bsz with UVOT on board the Neil Gehrels Swift Observatory. The UVOT photometry was performed using the task uvotsource in HEASoft ${ }^{4}$ version 6.25 , with a $3^{\prime \prime}$-radius aperture. For host subtraction, a template was constructed from images obtained between MJD $=58562$ and 59068 . We measured the host contribution using the same source and background apertures and numerically subtracted the host contribution from the SN light curve. While this manuscript was in preparation, a new version of the Swift CALDB was released which affects the zeropoint for our UVOT data. However, as the change in photometry is less than 0.1 mag and within the photometric uncertainties in all epochs, we have not implemented this change here and it will not affect our results.

4 https://heasarc.gsfc.nasa.gov/docs/software/heasoft/ 


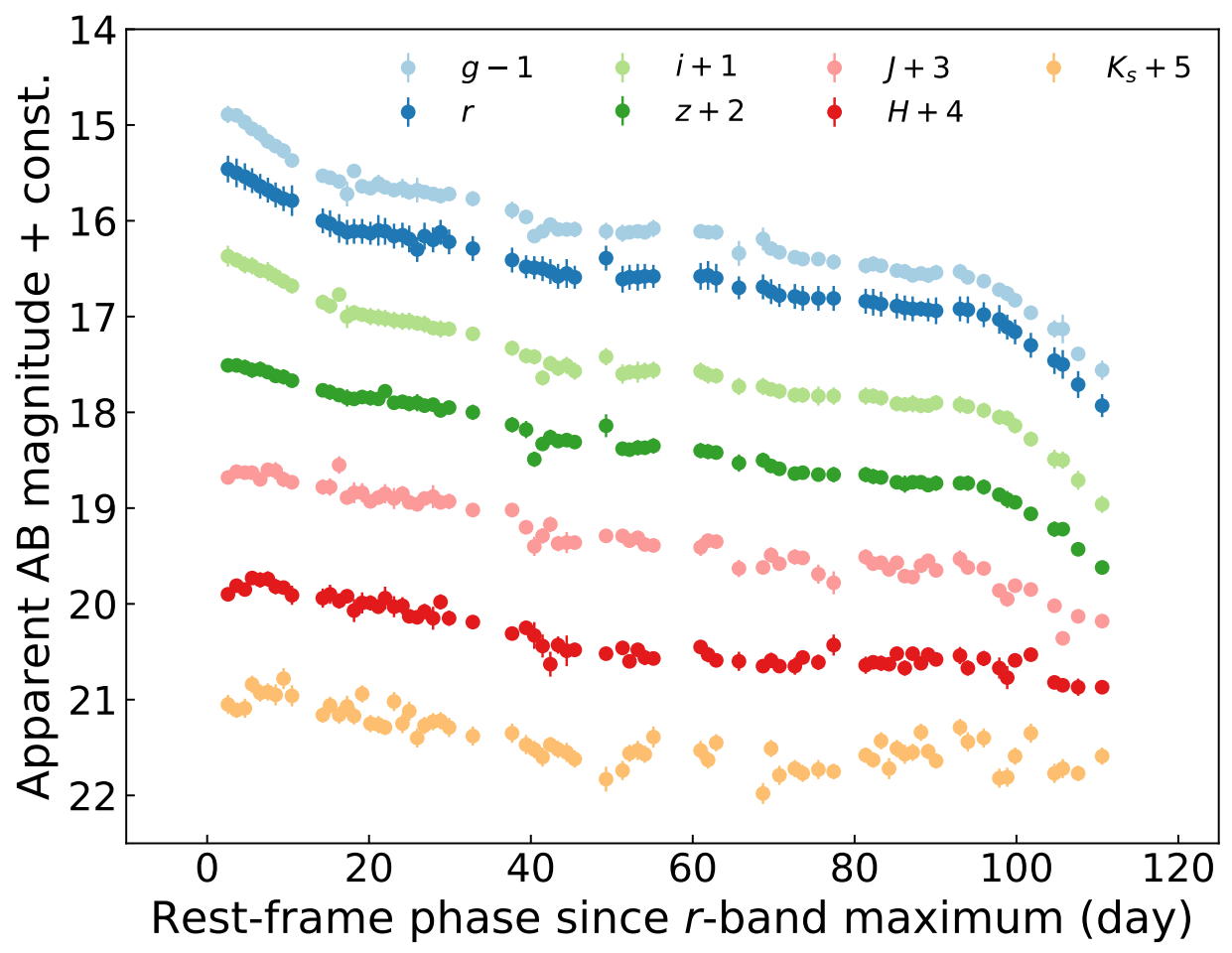

Figure A2. GROND host-subtracted light curves of SN 2018bsz. These data are the first daily-cadence and simultaneous 7-band observations of an SLSN.

\section{A.2. Optical and near-infrared imaging from the ground}

We used GROND to monitor the light-curve evolution of SN 2018bsz from $+3 \mathrm{~d}$ to $+421 \mathrm{~d}$. This 7 -channel imager collects multi-band photometry simultaneously in the $g^{\prime}, r^{\prime}, i^{\prime}, z^{\prime}, J, H$ and $K_{\mathrm{s}}$ bands. The images were reduced using the GROND pipeline (Krühler et al. 2008), which applies de-bias and flat-field corrections, stacks images and provides astrometric calibration. For GROND NIR images, due to the bright host galaxy we disabled line by line fitting of the sky subtraction routine since this caused over-subtraction artifacts.

All photometric measurements of the imaging data were carried out using the custom-built photometry pipeline AutoPhOT $^{5}$ (Brennan et al. 2021). Point spread function (PSF) photometry was performed using a PSF model built from bright, isolated sources in the image. We adopted the $+610 \mathrm{~d}$ epoch as the host galaxy template and subtracted that before measuring the SN brightness using AutoPhOT. The optical magnitudes are calibrated against PanSTARRS field stars while NIR images are calibrated to 2MASS and converted to the AB system. The PSF model for each image is used to determine limiting magnitudes. We collect a random sample of pixels within a cutout around SN 2018bsz and build a pseudo-PSF model. We then assert a confidence that pixels at the transient are real detection, rather than a correlated noise spike.

For this work, limiting magnitudes are set to $3 \sigma$ confidence. For further details, see Brennan et al. (2021). Figure A2 shows our GROND light curves of SN 2018bsz from $+3 \mathrm{~d}$ to $+111 \mathrm{~d}$.

In addition, we obtained several $J$ and $K_{\mathrm{s}}$-band images using SOFI on NTT through the ePESSTO program. The images were reduced using the PESSTO pipeline (Smartt et al. 2015) with flat-fielding and sky background subtraction. The host templates were taken around $+600 \mathrm{~d}$ and we measured the host-subtracted photometry of those SOFI images using AutoPhOT.

\footnotetext{
${ }^{5}$ https://github.com/Astro-Sean/autophot
} 


\section{A.3. Mid-infrared imaging from space}

We obtained Spitzer observations at four different epochs between 2019 June 27 (at epoch +384 d) and 2019 December $30(+564 \mathrm{~d})$, just before the end of the Spitzer mission. The images were taken using IRAC in the 3.6 and $4.5 \mu \mathrm{m}$ bands, which we here refer to as the $C h 1$ and $C h 2$ bands.

We aligned the images using the GEOMAP and GEOTRAN tasks within IRAF and employed the High Order Transform of PSF ANd Template Subtraction (hotpants) ${ }^{6}$ package to carry out the host template subtraction. We used the last epoch as the host template and subtracted the other frames from it. The image subtraction results are shown in Fig. A6. We measured the counts from the SN in those host-subtracted images using the DAOPHOT task within IRAF. With an aperture size of 6.1 pixel radius, a conversion factor of $8.461595 \times 10^{-6}$ and a aperture correction of 1.112 , we calculated the SN fluxes in Janskys and converted them to magnitudes. More details on conversion factor and aperture correction can be found from the Spitzer Heritage Archive ${ }^{7,8}$. The errors were dominated by the standard deviation of the sky background pixels around the SN positions.

The Spitzer $m_{C h 1}$ measurement at $+384 \mathrm{~d}$ is consistent with the evolution of the NEOWISE $m_{W 1}$ magnitudes. This validate our choice to use the $+565 \mathrm{~d}$ epoch as a template, because the NEOWISE magnitudes are obtained by subtracting a template that was obtained before the SN explosion. On the other hand, assuming a linear decline from the epoch at $+384 \mathrm{~d}$ to $+403 \mathrm{~d}$, the extrapolated magnitudes that we derived at $+535 \mathrm{~d}$ are $m_{C h 1} \sim 22.4$, $m_{C h 2} \sim 21.4 \mathrm{mag}$, respectively. Those are consistent within the uncertainties of the measured Spitzer magnitudes that result from using $+565 \mathrm{~d}$ epoch as the template $\left(m_{C h 1}=21.8 \pm 0.7, m_{C h 2}=21.6 \pm 0.6 \mathrm{mag}\right)$.

The NEOWISE-R data set consists of 12 epochs at $W 1(3.4 \mu \mathrm{m})$ and $W 2(4.6 \mu \mathrm{m})$ at a $\sim 6$ month cadence, with the final three epochs obtained after the discovery of SN 2018bsz. Each epoch consists of 12-18 individual exposures across $\sim 2$ days, with PSF-fitted magnitudes reported in the NEOWISE-R catalogue. An unresolved source was reported at the position of the host of SN 2018bsz at all epochs. We calculated the average magnitude at each epoch, where exposures flagged for poor quality were excluded, and the error was taken as the standard error of the mean combined with a flux error term to reflect the uncertainty between epochs (e.g., Kool et al. 2020). The nine NEOWISE-R epochs up until the SN discovery did not show significant evolution within the errors at $W 1$ and $W 2$ bands. Based on these nine epochs we determined mean quiescent magnitudes at $W 1$ and $W 2$ bands of the host of SN 2018bsz of $13.50 \pm 0.01$ and $13.32 \pm 0.02 \mathrm{mag}$, respectively. The mean quiescent magnitudes were then subtracted, in flux space, from the three post-SN NEOWISE-R epochs to obtain the contribution of the SN at $W 1$ and $W 2$ bands at each epoch. The final SN magnitude errors include the statistical error and the systematic error of the quiescent magnitude. More details on this method are described in Kool et al. (2020).

\section{B. OPTICAL AND NIR SPECTROSCOPIC OBSERVATIONS OF SN 2018BSZ}

We obtained a series of spectra of SN 2018bsz using different instruments (a log of observations is given in Table 1).

SINFONI is a NIR IFU fed by an adaptive optics (AO) module. We used the SN itself as a natural guide star, in order to perform an AO corrections. Unfortunately as SN 2018bsz was around mag 16 at the time these observations were taken it was too faint to obtain a satisfactory AO correction. The observations hence have a seeing $\sim 1^{\prime \prime}$, much larger than expected.

WiFeS is an IFU with 25 slitlets that are $1^{\prime \prime}$ wide and $38^{\prime \prime}$ long. The data were reduced using a custom-built pipeline PyWiFeS (Childress et al. 2014). The pre-peak NTT+EFOSC2 spectrum was taken from Anderson et al. (2018); the post-peak spectrum at $+108 \mathrm{~d}$ was reduced in the standard fashion using the PESSTO pipeline (Smartt et al. 2015).

The X-Shooter data were reduced using PYPEIT ${ }^{9}$ package (Prochaska et al. 2020; Prochaska et al. 2020). However, no SN trace is detected, and in particular we see no clear broad feature at the red extremity of the spectrum where we expect $\mathrm{CO}$ emission $(\sim 23,000 \AA)$.

At +417 d, when SN 2018zd had disappeared in the optical we used MUSE to observe the host environment. The data were reduced and combined into single MUSE data cubes using the standard ESO pipeline software version 1.2.11 (Weilbacher et al. 2014). We also subtracted the sky lines from our data cubes using the Zurich Atmospheric Purge (ZAP; v2.0 Soto et al. 2016) software and corrected for telluric absorptions using the molecfit software package (Smette et al. 2015). Detailed data reduction procedures are described in Schady et al. (2019).

\footnotetext{
${ }^{6}$ http://www.astro.washington.edu/users/becker/v2.0/hotpants.html

7 https://irsa.ipac.caltech.edu/data/SPITZER/docs/irac/iracinstrumenthandbook/24/

8 https://irsa.ipac.caltech.edu/data/SPITZER/docs/irac/iracinstrumenthandbook/27/\#_Toc410728317

${ }^{9}$ https://pypeit.readthedocs.io/en/latest/
} 


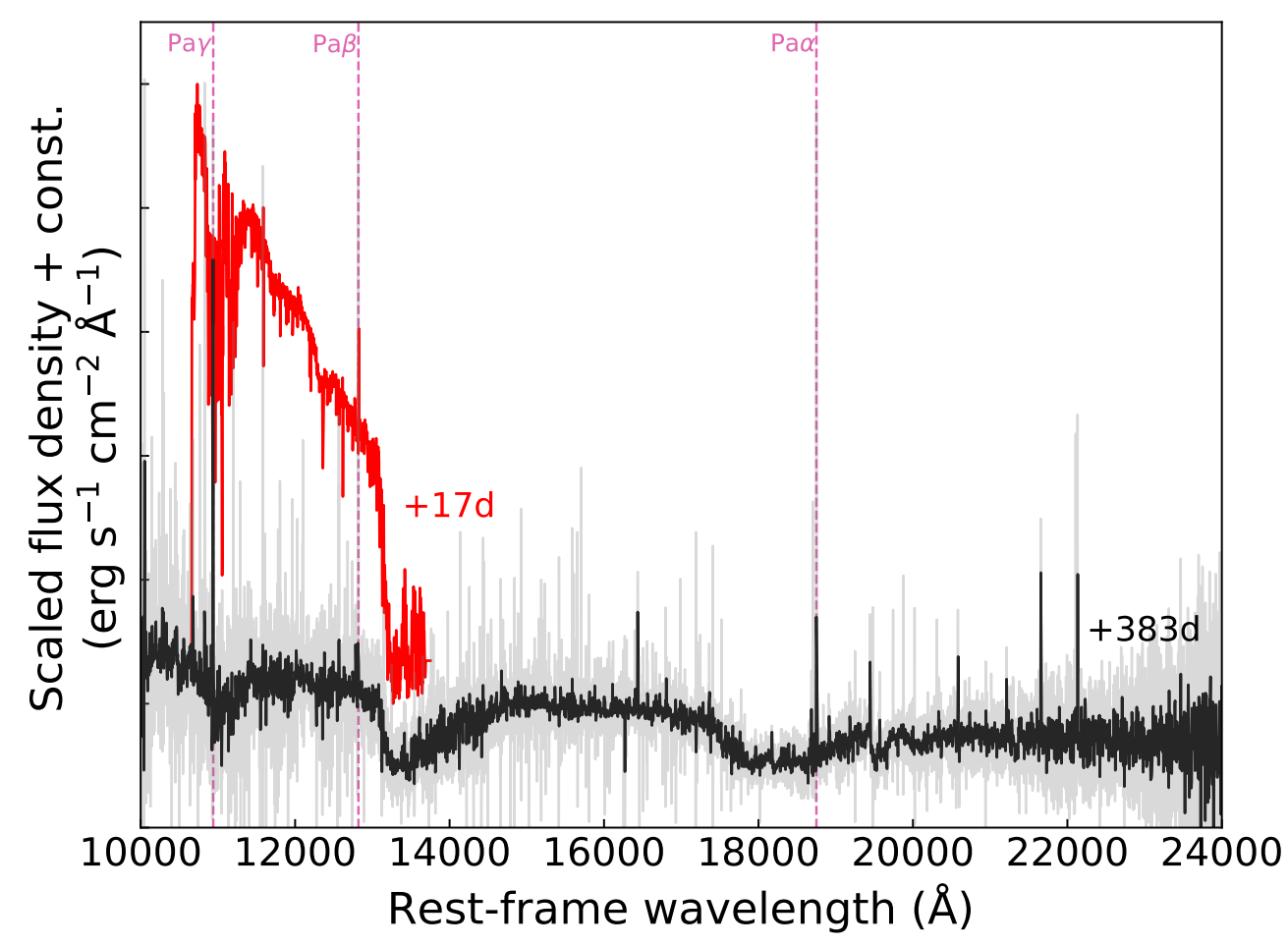

Figure A3. NIR spectra of SN 2018bsz. We extracted the SN position in the SINFONI data cube obtained at $+17 \mathrm{~d}$. A narrow $\mathrm{Pa} \beta$ is clearly detected, which is from the host $\mathrm{H}$ in region. No broad emission from the SN has been seen, confirming the hydrogen poor ejecta at peak brightness. The X-Shooter spectrum was taken on $+383 \mathrm{~d}$, no trace from the SN been detected.

We measured the emission line fluxes from the extracted MUSE spectrum at the SN position. The spectrum has been corrected for the Milky Way dust extinction and host dust extinction based on the Balmer decrement, and the stellar continuum has been subtracted. The flux and uncertainty measurement follow the method in Chen et al. (2017b). We adopted the Pettini \& Pagel (2004) calibration of the N2 and O3N2 methods, which use the $\log ([\mathrm{N} \mathrm{II}] \lambda 6583 / \mathrm{H} \alpha)$ ratio, giving $12+\log (\mathrm{O} / \mathrm{H})=8.27 \pm 0.06$; the $\log ([\mathrm{O} \mathrm{III}] \lambda 5007 / \mathrm{H} \beta) /([\mathrm{N} \mathrm{II}] \lambda 6583 / \mathrm{H} \alpha)$ ratio, giving $12+\log (\mathrm{O} / \mathrm{H})=8.27 \pm 0.05$, respectively. Moreover, we also adopted a metallicity diagnostic of Dopita et al. (2016), which uses [N II] $66583, \mathrm{H} \alpha,[\mathrm{S} \mathrm{II}] \lambda \lambda 6717,6731$ lines, giving $12+\log (\mathrm{O} / \mathrm{H})=8.14 \pm 0.10$. Assuming a solar oxygen abundance of $12+\log (\mathrm{O} / \mathrm{H})_{\odot}=8.69$ (Asplund et al. 2009), we found the local metallicity at the position of SN 2018bsz to be $\approx 0.3-0.4 Z_{\odot}$.

\section{BOLOMETRIC LIGHT-CURVE CONSTRUCTION}

We used the Superbol code ${ }^{10}$ (Nicholl 2018) to construct the bolometric light curve of SN 2018bsz. We integrated the flux for SN 2018bsz over the observed bands from UV to MIR between $+2 \mathrm{~d}$ and $+265 \mathrm{~d}$, which gives very similar results to the bolometic lightcurve computed using blackbody extrapolations. We used the $K_{\mathrm{s}}$-band epochs as our reference passband and interpolated and extrapolated the other bands by fitting a low-order polynomial to estimate the magnitude at epochs with missing data in a given filter. Unfortunately, during the pre-peak rising phase, we only have the optical light curve (with two colours), and thus we used the $o$ band as the reference filter here. We assumed that the colours are the same as that at peak brightness to extrapolate the UV, other optical bands and NIR. For the MIR contribution, we smoothly interpolated the MIR magnitudes between the $W$ ISE bands $+68 \mathrm{~d}$ and $+265 \mathrm{~d}$. For earlier phases after peak, we run SUPERBOL again with no MIR data, and added a constant fraction to all epochs based on the MIR fraction at $+68 \mathrm{~d}$ of $1 \%$. Finally we assumed no MIR contribution before the peak. We show the

10 https://github.com/mnicholl/superbol/blob/master/superbol.py 


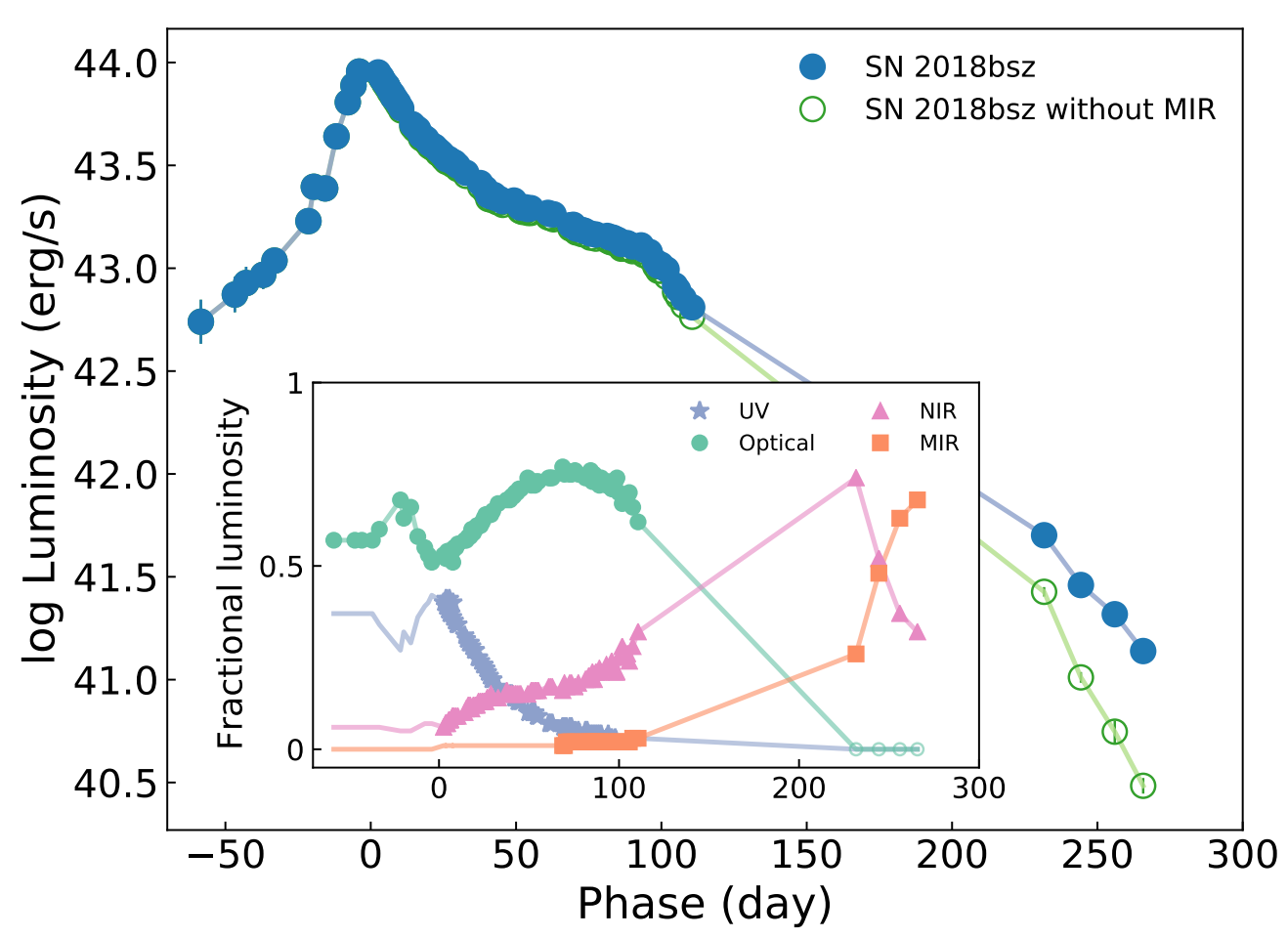

Figure A4. The bolometric light curve of SN 2018bsz. The blue solid points show the lightcurve integrated over all bands as discussed in the text, while the empty green points show the integration excluding the MIR contribution. The inset panel shows the relative contribution of UV (uvw2, uvm2, uvw1), optical $(U, B, g, c, V, r, o, i, z), \mathrm{NIR}\left(J, H, K_{s}\right)$ and MIR (W1, W2).

bolometric light curves with and without MIR contribution in Fig. A4, those are basically the same for the first 150 days as the MIR contribution is negligible in the early time.

The inset in Figure A4 shows the fractional luminosity in different wavelength ranges for SN 2018bsz. During the peak luminosity, the fraction of flux in the UV (uvw2, uvm2, uvw1; 1597-4730 $\AA$ ) is $40 \%$, optical $(U, B, g, c, V, r, o, i, z$; $3018-10647 \AA)$ is $53 \%$, NIR $\left(J, H, K_{\mathrm{s}} ; 10707-24537 \AA\right.$ ) is $6 \%$ and MIR $(W 1, W 2 ; 2.75-5.34 \mu \mathrm{m})$ is only $1 \%$ (assuming a constant fraction based on that at $+68 \mathrm{~d}$ ). After the peak, the UV fraction starts to decline, the optical fraction starts to increase and reaches $76 \%$ around $+75 \mathrm{~d}$, and the NIR fraction slowly increases. At $+40 \mathrm{~d}$, the fraction from the UV and the NIR was equal, both contributing $15 \%$ to the total luminosity. At $+111 \mathrm{~d}$, the fraction of UV dropped to $3 \%$, the optical is $62 \%$, the NIR raised to $32 \%$, and the MIR is $3 \%$. At the late-time phase of $+232 \mathrm{~d}$, there is no contribution from the UV and optical, the fraction from the NIR dominates at $74 \%$, and the MIR is $26 \%$. Just 34 days later the MIR has significantly increased to $68 \%$.

\section{OBSERVATION LOG}



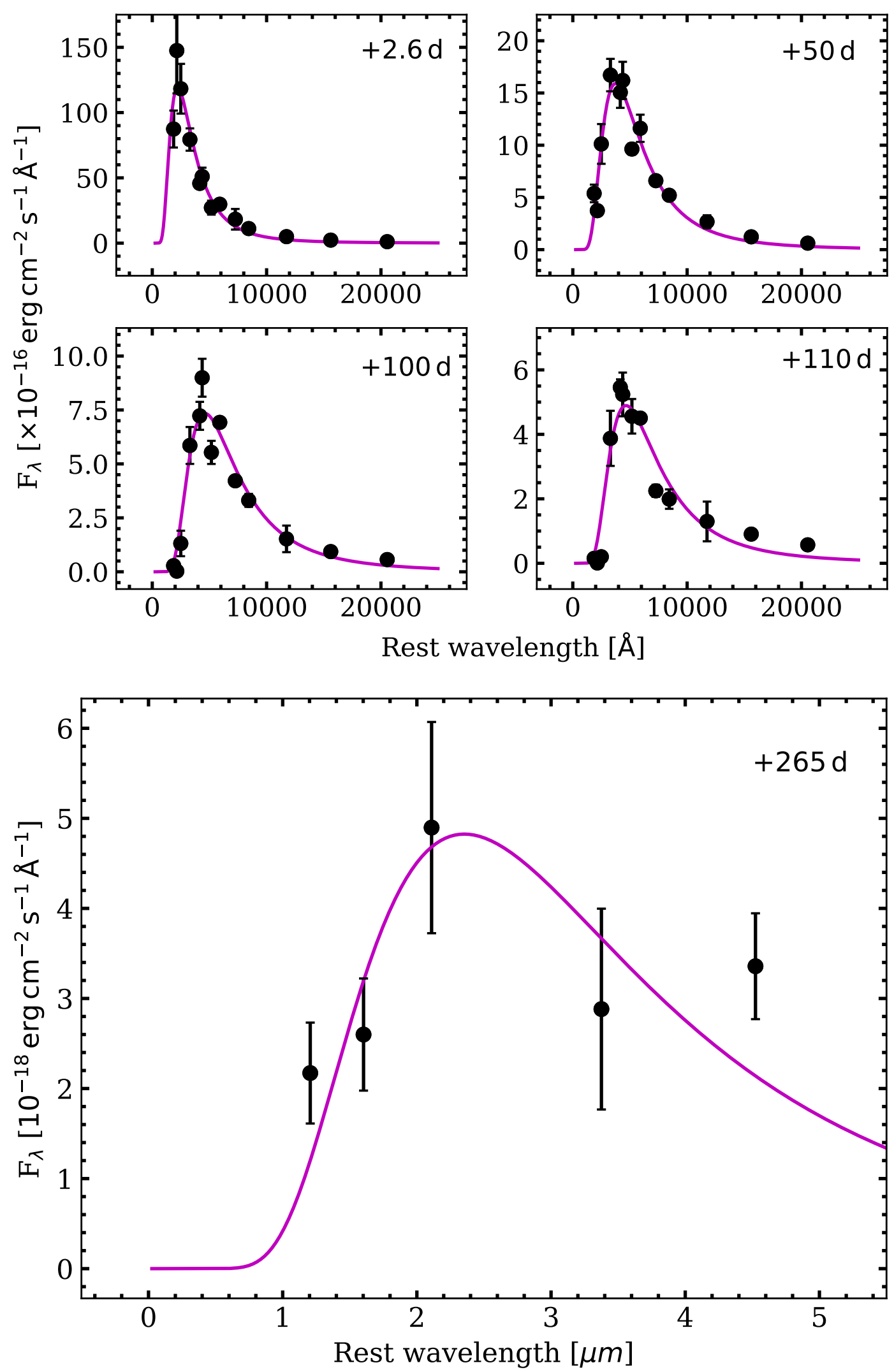

Figure A5. SED fitting for SN 2018bsz at selected epochs at $+2.6,+50,+100,+110$ and +265 d. The fluxes were converted from the observed magnitudes after correcting for Milky Way and host extinction. We find a single blackbody can fit the observed SED well for all selected epochs and do not require two distinct components to fit the SED. 


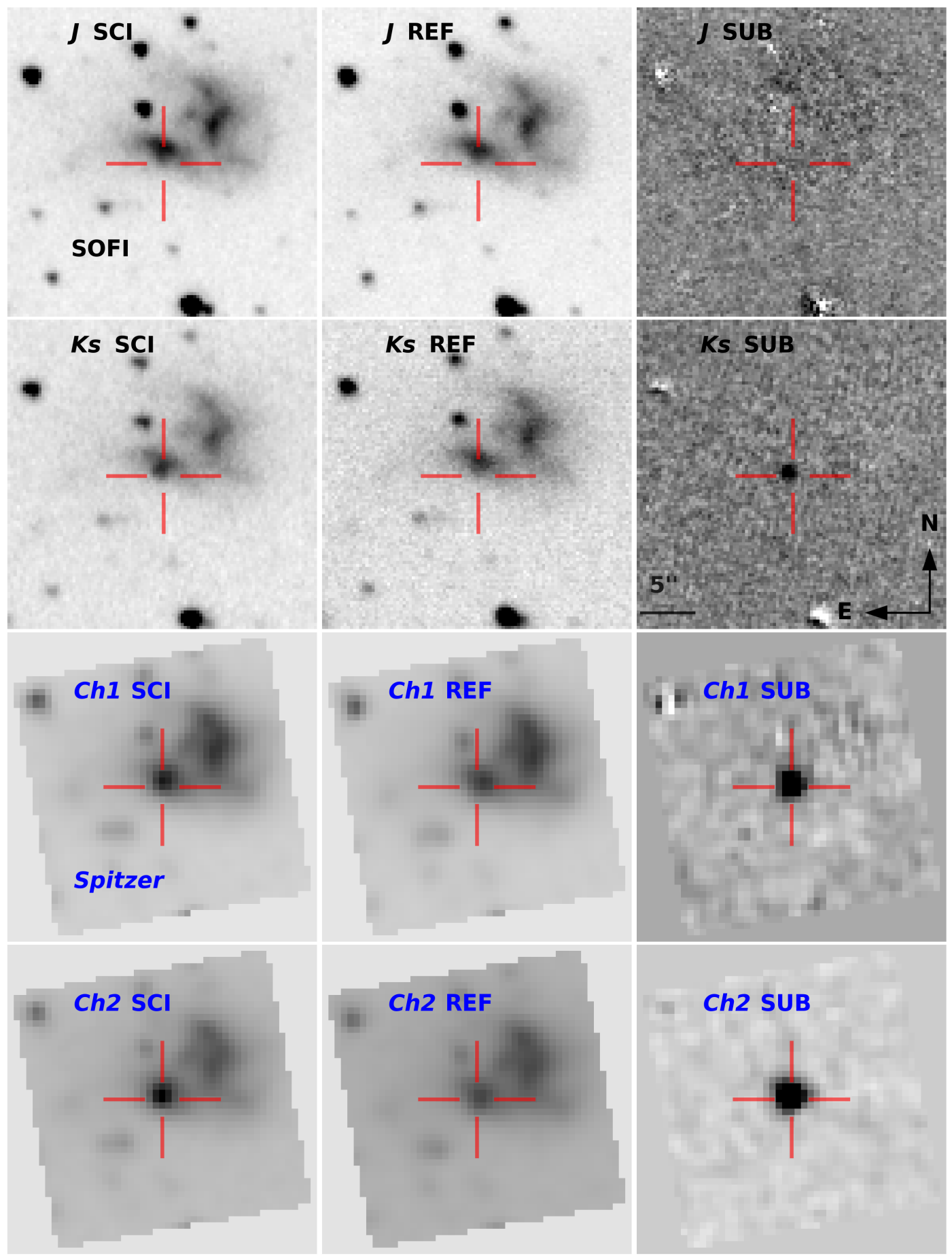

Figure A6. SOFI and Spitzer images of SN 2018bsz and template subtractions. The first row shows SOFI $J$-band frame taken on $+266 \mathrm{~d}$, the reference image taken on $+599 \mathrm{~d}$, and the subtraction between the two (with a $3 \sigma$ detection limit of $>21.92 \mathrm{mag}$ ). The second row shows SOFI $K_{\mathrm{s}}$-band images, as the same epoch as in the first row. The $K_{\mathrm{s}}$-band subtraction clearly shows a source at $+266 \mathrm{~d}$ where we detect the $\mathrm{SN}$ of $19.65 \pm 0.15 \mathrm{mag}$. The third row shows Spitzer Ch1 (3.6 $\mu \mathrm{m})$ frame taken on $+384 \mathrm{~d}$, the reference image taken on $+565 \mathrm{~d}$, and subtraction result with a SN detection at $20.17 \pm 0.24$ mag. The fourth row shows the Spitzer $C h 2(4.5 \mu \mathrm{m})$ images, at the same epoch as in the third row, where the SN was detected at $19.13 \pm 0.09 \mathrm{mag}$. Each panel has the same scale and orientation as marked in the second row. 
Table 1. Spectroscopic observations.

\begin{tabular}{|c|c|c|c|c|c|c|c|c|c|}
\hline Date & MJD & $\begin{array}{l}\text { Phase } \\
\text { (day) }\end{array}$ & Telescope & Instrument & Grism or Grating & $\begin{array}{c}\text { Exp. time } \\
\text { (s) }\end{array}$ & $\begin{array}{l}\text { Slit } \\
\left({ }^{\prime \prime}\right)\end{array}$ & $\begin{array}{c}\text { Resolution } \\
(\AA)\end{array}$ & $\begin{array}{c}\text { Range } \\
(\AA)\end{array}$ \\
\hline 2018-05-23 & 58261.290 & -6 & NTT & EFOSC2 & $\mathrm{Gr} \# 11 / \mathrm{Gr} \# 16$ & $1500 / 1500$ & $1.0 / 1.0$ & $13.8 / 13.4$ & $3400-7400 / 6000-9900^{a}$ \\
\hline 2019-06-16 & 58285 & +17 & VLT & SINFONI & $\mathrm{J}$ & 6300 & IFU & & $11,000-14,000$ \\
\hline 2018-08-16 & 58346.561 & +77 & ANU $2.3-\mathrm{m}$ & WiFeS & B3000/R3000 & $1800 / 1800$ & IFU & $1.6 / 2.5$ & $3500-5700 / 5400-9500$ \\
\hline 2018-09-17 & 58378.035 & +108 & NTT & EFOSC2 & $\mathrm{Gr} \# 11 / \mathrm{Gr} \# 16$ & $2700 / 2700$ & $1.0 / 1.0$ & $13.8 / 13.4$ & 3400-7400/6000-9900 \\
\hline 2019-04-30 & 58603 & +327 & VLT & X-Shooter & UVB/VIS/NIR & $3600 / 3712 / 3600$ & $0.9 / 0.9 / 1.0$ & $1 / 1.1 / 3.3$ & $3000-5560 / 5450-10,200 / 10,000-20,600$ \\
\hline 2019-05-05 & 58608 & +332 & VLT & X-Shooter & UVB/VIS/NIR & $7200 / 7424 / 7200$ & $0.9 / 0.9 / 1.0$ & $1 / 1.1 / 3.3$ & $3000-5560 / 5450-10,200 / 10,000-20,600$ \\
\hline 2019-05-06 & 58609 & +333 & VLT & X-Shooter & UVB/VIS/NIR & $3600 / 3712 / 3600$ & $0.9 / 0.9 / 1.0$ & $1 / 1.1 / 3.3$ & $3000-5560 / 5450-10,200 / 10,000-20,600$ \\
\hline 2019-06-27 & 58661 & +383 & VLT & X-Shooter & UVB/VIS/NIR & $7200 / 7424 / 7200$ & $0.9 / 0.9 / 1.0$ & $1 / 1.1 / 3.3$ & $3000-5560 / 5450-10,200 / 10,000-20,600$ \\
\hline 2019-08-01 & 58696 & +417 & VLT & MUSE & & 2139 & IFU & & $4650-9300$ \\
\hline
\end{tabular}

Note-a: the $-6 \mathrm{~d}$ spectrum is taken from Anderson et al. 2018. b: the $+108 \mathrm{~d}$ spectrum will be presented in Pursiainen et al. in prep.

Table 2. Blackbody temperature and radius evolution at selected epochs.

\begin{tabular}{ccc}
\hline \hline Phase & Temperature & Radius \\
$($ day $)$ & $(\mathrm{K})$ & $(\mathrm{cm})$ \\
\hline+2.6 & $13050(630)$ & $1.70(0.20) \times 10^{14}$ \\
+50 & $7760(230)$ & $2.30(0.20) \times 10^{15}$ \\
+100 & $6295(255)$ & $2.60(0.30) \times 10^{15}$ \\
+110 & $6230(280)$ & $2.20(0.25) \times 10^{15}$ \\
+265 & $1230(110)$ & $1.25(0.30) \times 10^{16}$ \\
\hline
\end{tabular}




\section{REFERENCES}

Anderson, J. P., Pessi, P. J., Dessart, L., et al. 2018, A\&A, 620, A67

Andrews, J. E., Gallagher, J. S., Clayton, G. C., et al. 2010, ApJ, 715, 541

Andrews, J. E., Sugerman, B. E. K., Clayton, G. C., et al. 2011, ApJ, 731, 47

Andrews, J. E., Krafton, K. M., Clayton, G. C., et al. 2016, MNRAS, 457, 3241

Angus, C. R., Levan, A. J., Perley, D. A., et al. 2016, MNRAS, 458, 84

Angus, C. R., Smith, M., Sullivan, M., et al. 2019, MNRAS, 487, 2215

Arnett, D., Fryxell, B., \& Mueller, E. 1989, ApJL, 341, L63

Asplund, M., Grevesse, N., Sauval, A. J., \& Scott, P. 2009, ARA\&A, 47, 481

Bacon, R., Accardo, M., Adjali, L., et al. 2010, in Society of Photo-Optical Instrumentation Engineers (SPIE)

Conference Series, Vol. 7735, Ground-based and Airborne Instrumentation for Astronomy III, ed. I. S. McLean, S. K. Ramsay, \& H. Takami, 773508

Barlow, M. J., Sugerman, B. E. K., Fabbri, J., et al. 2005, ApJL, 627, L113

Bertoldi, F., Carilli, C. L., Cox, P., et al. 2003, A\&A, 406, L55

Bevan, A., \& Barlow, M. J. 2016, MNRAS, 456, 1269

Bevan, A., Barlow, M. J., \& Milisavljevic, D. 2017, MNRAS, 465, 4044

Bevan, A., Wesson, R., Barlow, M. J., et al. 2019, MNRAS, 485, 5192

Bevan, A. M., Krafton, K., Wesson, R., et al. 2020, ApJ, 894, 111

Bhirombhakdi, K., Chornock, R., Miller, A. A., et al. 2019, MNRAS, 488, 3783

Bian, F., Kewley, L. J., Dopita, M. A., \& Juneau, S. 2016, ApJ, 822, 62

Bianchi, S., \& Schneider, R. 2007, MNRAS, 378, 973

Botticella, M. T., Trundle, C., Pastorello, A., et al. 2010, ApJL, 717, L52

Brennan, S. J., Fraser, M., Johansson, J., et al. 2021, arXiv e-prints, arXiv:2102.09572

Buzzoni, B., Delabre, B., Dekker, H., et al. 1984, The Messenger, 38, 9

Calura, F., Gilli, R., Vignali, C., et al. 2014, MNRAS, 438, 2765

Cardamone, C., Schawinski, K., Sarzi, M., et al. 2009, MNRAS, 399, 1191

Cardelli, J. A., Clayton, G. C., \& Mathis, J. S. 1989, ApJ, 345,245
Cernuschi, F., Marsicano, F., \& Codina, S. 1967, Annales d'Astrophysique, 30, 1039

Chen, T.-W., Smartt, S. J., Yates, R. M., et al. 2017a, MNRAS, 470, 3566

Chen, T.-W., Smartt, S. J., Bresolin, F., et al. 2013, ApJL, $763, \mathrm{~L} 28$

Chen, T.-W., Smartt, S. J., Jerkstrand, A., et al. 2015, MNRAS, 452, 1567

Chen, T.-W., Nicholl, M., Smartt, S. J., et al. 2017b, A\&A, 602, A9

Chen, T. W., Inserra, C., Fraser, M., et al. 2018, ApJL, 867, L31

Cherchneff, I., \& Dwek, E. 2009, ApJ, 703, 642

—. 2010, ApJ, 713, 1

Childress, M. J., Vogt, F. P. A., Nielsen, J., \& Sharp, R. G. 2014, Ap\&SS, 349, 617

Cid Fernandes, R., Mateus, A., Sodré, L., Stasińska, G., \& Gomes, J. M. 2005, MNRAS, 358, 363

Clayton, D. D., Deneault, E. A. N., \& Meyer, B. S. 2001, ApJ, 562, 480

Cooke, J., Sullivan, M., Gal-Yam, A., et al. 2012, Nature, 491,228

Curtin, C., Cooke, J., Moriya, T. J., et al. 2019, ApJS, 241, 17

Danziger, I. J., Gouiffes, C., Bouchet, P., \& Lucy, L. B. 1989, IAUC, 4746, 1

Davis, S., Hsiao, E. Y., Ashall, C., et al. 2019, ApJ, 887, 4

De Looze, I., Barlow, M. J., Swinyard, B. M., et al. 2017, MNRAS, 465, 3309

Dopita, M., Hart, J., McGregor, P., et al. 2007, Ap\&SS, 310,255

Dopita, M. A., Kewley, L. J., Sutherland, R. S., \& Nicholls, D. C. 2016, Ap\&SS, 361, 61

Douvion, T., Lagage, P. O., \& Pantin, E. 2001, A\&A, 369, 589

Dwek, E. 1998, ApJ, 501, 643

Dwek, E., \& Arendt, R. G. 2015, ApJ, 810, 75

Dwek, E., \& Cherchneff, I. 2011, ApJ, 727, 63

Dwek, E., Galliano, F., \& Jones, A. P. 2007, ApJ, 662, 927

Eisenhauer, F., Abuter, R., Bickert, K., et al. 2003, in Society of Photo-Optical Instrumentation Engineers (SPIE) Conference Series, Vol. 4841, Instrument Design and Performance for Optical/Infrared Ground-based Telescopes, ed. M. Iye \& A. F. M. Moorwood, 1548-1561

Elias, J. H., Gregory, B., Phillips, M. M., et al. 1988, ApJL, 331, L9

Elmhamdi, A., Danziger, I. J., Cappellaro, E., et al. 2004, A\&A, 426, 963 
Elmhamdi, A., Danziger, I. J., Chugai, N., et al. 2003, MNRAS, 338, 939

Ercolano, B., Barlow, M. J., \& Storey, P. J. 2005, MNRAS, 362,1038

Ercolano, B., Barlow, M. J., Storey, P. J., \& Liu, X. W. 2003, MNRAS, 340, 1136

Ercolano, B., Barlow, M. J., \& Sugerman, B. E. K. 2007, MNRAS, 375, 753

Fazio, G. G., Hora, J. L., Allen, L. E., et al. 2004, ApJS, 154,10

Ferrarotti, A. S., \& Gail, H. P. 2006, A\&A, 447, 553

Fox, O. D., Chevalier, R. A., Dwek, E., et al. 2010, ApJ, 725,1768

Frohmaier, C., Angus, C. R., Vincenzi, M., et al. 2021, MNRAS, 500, 5142

Gal-Yam, A. 2019, ARA\&A, 57, 305

Gal-Yam, A., Mazzali, P., Ofek, E. O., et al. 2009, Nature, 462, 624

Gall, C., Andersen, A. C., \& Hjorth, J. 2011a, A\&A, 528, A14

Gall, C., Hjorth, J., \& Andersen, A. C. 2011b, A\&A Rv, 19,43

Gall, C., Hjorth, J., Watson, D., et al. 2014, Nature, 511, 326

Gallagher, J. S., Sugerman, B. E. K., Clayton, G. C., et al. 2012, ApJ, 753, 109

Gehrels, N., Chincarini, G., Giommi, P., et al. 2004, ApJ, 611,1005

Gezari, S., Halpern, J. P., Grupe, D., et al. 2009, ApJ, 690, 1313

Gomez, H. L., Krause, O., Barlow, M. J., et al. 2012, ApJ, 760,96

Greiner, J., Bornemann, W., Clemens, C., et al. 2008, PASP, 120, 405

Gutiérrez, C. P., Anderson, J. P., Hamuy, M., et al. 2017, ApJ, 850, 90

Heckman, T. M., Hoopes, C. G., Seibert, M., et al. 2005, ApJL, 619, L35

Henriques, B. M. B., Yates, R. M., Fu, J., et al. 2020, MNRAS, 491, 5795

Hirashita, H., \& Ferrara, A. 2002, MNRAS, 337, 921

Hoyle, F., \& Wickramasinghe, N. C. 1970, Nature, 226, 62

Indebetouw, R., Matsuura, M., Dwek, E., et al. 2014, ApJL, 782, L2

Inserra, C. 2019, Nature Astronomy, 3, 697

Inserra, C., Baron, E., \& Turatto, M. 2012, MNRAS, 422, 1178

Inserra, C., Turatto, M., Pastorello, A., et al. 2011, MNRAS, 417, 261
Inserra, C., Smartt, S. J., Jerkstrand, A., et al. 2013, ApJ, 770,128

Inserra, C., Nichol, R. C., Scovacricchi, D., et al. 2018a, A\&A, 609, A83

Inserra, C., Smartt, S. J., Gall, E. E. E., et al. 2018b, MNRAS, 475, 1046

Inserra, C., Sullivan, M., Angus, C. R., et al. 2021, MNRAS, 504, 2535

Jerkstrand, A., Smartt, S. J., Inserra, C., et al. 2017, ApJ, 835,13

Kasen, D., \& Bildsten, L. 2010, ApJ, 717, 245

Kool, E. C., Reynolds, T. M., Mattila, S., et al. 2020, arXiv e-prints, arXiv:2006.01518

Kotak, R., Meikle, W. P. S., Farrah, D., et al. 2009, ApJ, 704,306

Krühler, T., Küpcü Yoldaş, A., Greiner, J., et al. 2008, ApJ, 685, 376

Lanz, L., Zezas, A., Brassington, N., et al. 2013, ApJ, 768, 90

Leloudas, G., Schulze, S., Krühler, T., et al. 2015, MNRAS, 449, 917

Lucy, L. B., Danziger, I. J., Gouiffes, C., \& Bouchet, P. 1989, Dust Condensation in the Ejecta of SN 1987 A, ed. G. Tenorio-Tagle, M. Moles, \& J. Melnick, Vol. 350, 164

Lunnan, R., Chornock, R., Berger, E., et al. 2015, ApJ, 804,90

Maeda, K., Nozawa, T., Sahu, D. K., et al. 2013, ApJ, 776, 5

Maguire, K., Di Carlo, E., Smartt, S. J., et al. 2010, MNRAS, 404, 981

Mainzer, A., Bauer, J., Grav, T., et al. 2011, ApJ, 731, 53

Mainzer, A., Bauer, J., Cutri, R. M., et al. 2014, ApJ, 792, 30

Maiolino, R., Schneider, R., Oliva, E., et al. 2004, Nature, 431,533

Mancini, M., Schneider, R., Graziani, L., et al. 2015, MNRAS, 451, L70

Matsuura, M., Barlow, M. J., Zijlstra, A. A., et al. 2009, MNRAS, 396, 918

Matsuura, M., Dwek, E., Meixner, M., et al. 2011, Science, 333,1258

Matsuura, M., Dwek, E., Barlow, M. J., et al. 2015, ApJ, 800,50

Mattila, S., Meikle, W. P. S., Lundqvist, P., et al. 2008, MNRAS, 389, 141

Mazzali, P. A., Moriya, T. J., Tanaka, M., \& Woosley, S. E. 2019, MNRAS, 484, 3451

McGregor, P. J., Hyland, A. R., \& Beresford, A. C. 1987, IAUC, 4468,2 
Meikle, W. P. S., Mattila, S., Gerardy, C. L., et al. 2006, ApJ, 649, 332

Meikle, W. P. S., Mattila, S., Pastorello, A., et al. 2007, ApJ, 665, 608

Meza, N., Prieto, J. L., Clocchiatti, A., et al. 2019, A\&A, 629, A57

Miller, A. A., Chornock, R., Perley, D. A., et al. 2009, ApJ, 690, 1303

Morgan, H. L., \& Edmunds, M. G. 2003, MNRAS, 343, 427

Moriya, T. J., Chen, T.-W., \& Langer, N. 2017, ApJ, 835, 177

Moriya, T. J., Sorokina, E. I., \& Chevalier, R. A. 2018, SSRv, 214, 59

Moriya, T. J., Tanaka, M., Yasuda, N., et al. 2019, ApJS, 241,16

Neill, J. D., Sullivan, M., Gal-Yam, A., et al. 2011, ApJ, 727,15

Nicholl, M. 2018, Research Notes of the American Astronomical Society, 2, 230

Nicholl, M., Smartt, S. J., Jerkstrand, A., et al. 2013, Nature, 502, 346

—. 2015, MNRAS, 452, 3869

Nicholl, M., Berger, E., Smartt, S. J., et al. 2016, ApJ, 826, 39

Nicholl, M., Blanchard, P. K., Berger, E., et al. 2020, Nature Astronomy, 4, 893

Nozawa, T., Kozasa, T., Umeda, H., Maeda, K., \& Nomoto, K. 2003, ApJ, 598, 785

Nozawa, T., Maeda, K., Kozasa, T., et al. 2011, ApJ, 736, 45

Omand, C. M. B., Kashiyama, K., \& Murase, K. 2019, MNRAS, 484, 5468

Ørum, S. V., Ivens, D. L., Strandberg, P., et al. 2020, A\&A, 643, A47

Osterbrock, D. E. 1989, Astrophysics of gaseous nebulae and active galactic nuclei

Pan, Y. C., Foley, R. J., Smith, M., et al. 2017, MNRAS, 470, 4241

Perley, D. A., Quimby, R. M., Yan, L., et al. 2016, ApJ, 830,13

Pettini, M., \& Pagel, B. E. J. 2004, MNRAS, 348, L59

Poznanski, D., Prochaska, J. X., \& Bloom, J. S. 2012, MNRAS, 426, 1465

Pozzo, M., Meikle, W. P. S., Fassia, A., et al. 2004, MNRAS, 352, 457

Prochaska, J. X., Hennawi, J. F., Westfall, K. B., et al. 2020, Journal of Open Source Software, 5, 2308

Prochaska, J. X., Hennawi, J., Cooke, R., et al. 2020, pypeit/PypeIt: Release 1.0.0, doi:10.5281/zenodo.3743493
Quimby, R. M., Kulkarni, S. R., Kasliwal, M. M., et al. 2011, Nature, 474, 487

Rawlings, J. M. C., \& Williams, D. A. 1990, MNRAS, 246, 208

Rho, J., Geballe, T. R., Banerjee, D. P. K., et al. 2018, ApJL, 864, L20

Rho, J., Evans, A., Geballe, T. R., et al. 2021, ApJ, 908, 232

Roming, P. W. A., Kennedy, T. E., Mason, K. O., et al. 2005, SSRv, 120, 95

Sakon, I., Onaka, T., Wada, T., et al. 2009, ApJ, 692, 546

Sarangi, A., Dwek, E., \& Arendt, R. G. 2018a, ApJ, 859, 66

Sarangi, A., Matsuura, M., \& Micelotta, E. R. 2018b, SSRv, 214, 63

Schady, P., Eldridge, J. J., Anderson, J., et al. 2019, MNRAS, 490, 4515

Schlafly, E. F., \& Finkbeiner, D. P. 2011, ApJ, 737, 103

Schulze, S., Krühler, T., Leloudas, G., et al. 2018, MNRAS, 473,1258

Smartt, S. J., Valenti, S., Fraser, M., et al. 2015, A\&A, 579, A40

Smette, A., Sana, H., Noll, S., et al. 2015, A\&A, 576, A77

Smith, M., Sullivan, M., Nichol, R. C., et al. 2018, ApJ, 854,37

Soto, K. T., Lilly, S. J., Bacon, R., Richard, J., \& Conseil, S. 2016, MNRAS, 458, 3210

Spergel, D. N., Bean, R., Doré, O., et al. 2007, ApJS, 170, 377

Stritzinger, M., Taddia, F., Fransson, C., et al. 2012, ApJ, 756,173

Sugerman, B. E. K., Ercolano, B., Barlow, M. J., et al. 2006, Science, 313, 196

Sugerman, B. E. K., Andrews, J. E., Barlow, M. J., et al. 2012, ApJ, 749, 170

Szalai, T., \& Vinkó, J. 2013, A\&A, 549, A79

Szalai, T., Vinkó, J., Balog, Z., et al. 2011, A\&A, 527, A61

Tartaglia, L., Pastorello, A., Sollerman, J., et al. 2020, A\&A, 635, A39

Tinyanont, S., Lau, R. M., Kasliwal, M. M., et al. 2019a, ApJ, 887, 75

Tinyanont, S., Kasliwal, M. M., Krafton, K., et al. 2019b, ApJ, 873, 127

Todini, P., \& Ferrara, A. 2001, MNRAS, 325, 726

Tomasella, L., Cappellaro, E., Fraser, M., et al. 2013, MNRAS, 434, 1636

Valiante, R., Schneider, R., Bianchi, S., \& Andersen, A. C. 2009, MNRAS, 397, 1661

Valiante, R., Schneider, R., Salvadori, S., \& Bianchi, S. 2011, MNRAS, 416, 1916 
Vijayan, A. P., Clay, S. J., Thomas, P. A., et al. 2019, MNRAS, 489, 4072

Vreeswijk, P. M., Leloudas, G., Gal-Yam, A., et al. 2017, ApJ, 835, 58

Wang, L., Mould, J., Baade, D., et al. 2019, BAAS, 51, 399

Weilbacher, P. M., Streicher, O., Urrutia, T., et al. 2014, in Astronomical Society of the Pacific Conference Series, Vol. 485, Astronomical Data Analysis Software and Systems XXIII, ed. N. Manset \& P. Forshay, 451

Werner, M. W., Roellig, T. L., Low, F. J., et al. 2004, ApJS, 154, 1
Wesson, R., Barlow, M. J., Matsuura, M., \& Ercolano, B. 2015, MNRAS, 446, 2089

Wesson, R., Barlow, M. J., Ercolano, B., et al. 2010, MNRAS, 403, 474

Wright, E. L., Eisenhardt, P. R. M., Mainzer, A. K., et al. 2010, AJ, 140, 1868

Yan, L., Quimby, R., Ofek, E., et al. 2015, ApJ, 814, 108

Yan, L., Lunnan, R., Perley, D. A., et al. 2017, ApJ, 848, 6

Yates, R. M., Henriques, B. M. B., Fu, J., et al. 2021, MNRAS, 503, 4474 